\title{
Dynamic testing of nonlinear vibrating structures using nonlinear normal modes
}

\author{
M. Peeters *, G. Kerschen, J.C. Golinval \\ Structural Dynamics Research Group, Department of Aerospace and Mechanical Engineering, University of Liège, 1 Chemin des Chevreuils (B52/3), \\ $B-4000$ Liège, Belgium
}

\section{A R T I C L E I N F O}

\section{Article history:}

Received 25 September 2009

Received in revised form

20 August 2010

Accepted 20 August 2010

Handling Editor: L.N. Virgin

Available online 19 September 2010

\begin{abstract}
A B S T R A C T
Modal testing and analysis is well-established for linear systems. The objective of this paper is to progress toward a practical experimental modal analysis (EMA) methodology of nonlinear mechanical structures. In this context, nonlinear normal modes (NNMs) offer a solid theoretical and mathematical tool for interpreting a wide class of nonlinear dynamical phenomena, yet they have a clear and simple conceptual relation to the classical linear normal modes (LNMs). A nonlinear extension of force appropriation techniques is developed in this study in order to isolate one single NNM during the experiments. With the help of time-frequency analysis, the energy dependence of NNM modal curves and their frequencies of oscillation are then extracted from the time series. The proposed methodology is demonstrated using two numerical benchmarks, a two-degree-of-freedom system and a planar cantilever beam with a cubic spring at its free end.
\end{abstract}

(c) 2010 Elsevier Ltd. All rights reserved.

\section{Introduction}

In the virtual prototyping era, dynamic testing remains an important step of the design of engineering structures, because the accuracy of finite element predictions can be assessed [1]. In this context, experimental modal analysis (EMA) is indubitably the most popular approach and extracts the modal parameters (i.e., the mode shapes, natural frequencies and damping ratios). The popularity of EMA stems from its great generality; modal parameters can describe the behavior of a system for any input type and any range of the input.

The techniques available today for EMA are really quite sophisticated and advanced: eigensystem realization algorithm [2], stochastic subspace identification method [3], polyreference least-squares complex frequency-domain method [4], to name a few. Modal identification of complex industrial structures with high modal density and large modal overlap is now within reach. Unification of the theoretical development of EMA algorithms was attempted in [5,6], which is another sign of the maturity of this research field.

While the common practice is to assume linear behavior, nonlinearity is a frequent occurrence in engineering applications and can drastically alter their behavior. For instance, in an aircraft, besides nonlinear fluid-structure interaction, typical nonlinearities include backlash and friction in control surfaces and joints, hardening nonlinearities in engine-to-pylon connections, and saturation effects in hydraulic actuators [7]. Satellites are other examples of aerospace applications where nonlinearity may significantly impact the dynamic behavior $[8,9]$.

\footnotetext{
* Corresponding author. Tel.: +3243664854; fax: +3243669505.

E-mail addresses: m.peeters@ulg.ac.be (M. Peeters), g.kerschen@ulg.ac.be (G. Kerschen), jc.golinval@ulg.ac.be (J.C. Golinval).
} 
Finite element model

$\mathbf{M} \ddot{\mathbf{x}}(t)+\mathbf{K} \mathbf{x}(t)+\mathbf{f}_{n l}\{\mathbf{x}(t)\}=0$
Experimental response (time series)
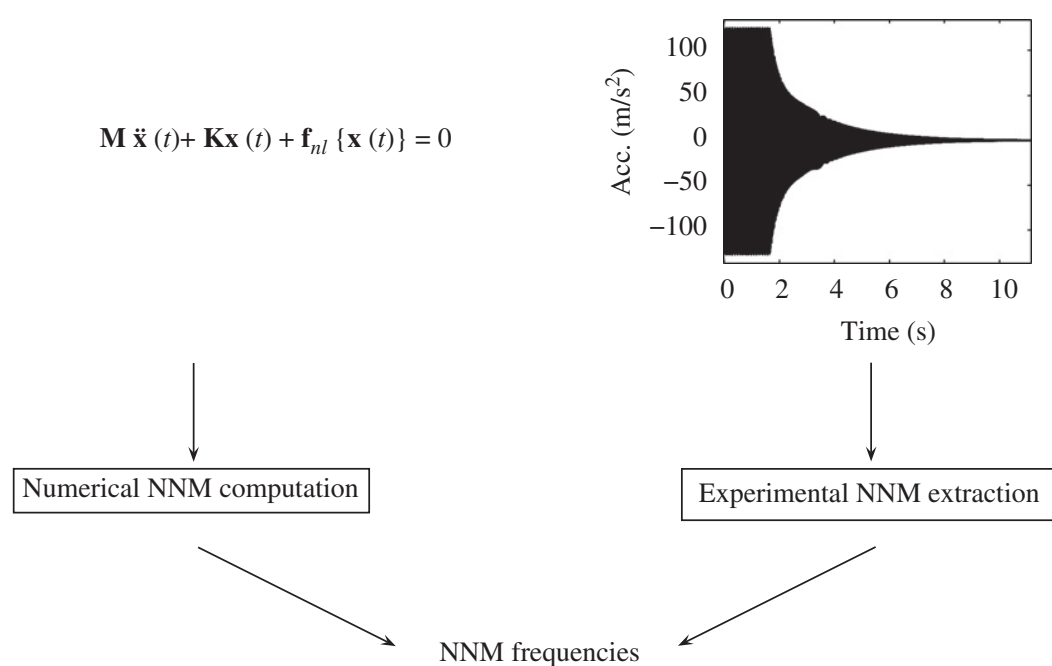

NNM frequencies

NNM modal curves
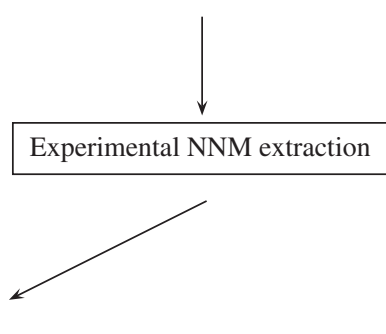

Fig. 1. Theoretical and experimental nonlinear modal analysis.

As reported in [10], a large body of literature exists regarding dynamic testing and identification of nonlinear structures, but very little work addresses nonlinear phenomena during modal survey tests. Interesting contributions in this context are [11-13]. The force appropriation of nonlinear systems (FANS) method extends linear force appropriation to nonlinear structures [11]. A multi-exciter force pattern that includes higher harmonic terms is used to counteract nonlinear coupling terms, which prevent any response other than the linear normal mode of interest. The nonlinear resonant decay (NLRD) method applies a burst of a sine wave at the undamped natural frequency of a linear mode and enables small groups of modes coupled by nonlinear forces to be excited [12]. A nonlinear curve fit in modal space is then carried out using the restoring force surface (RFS) method. Another test strategy that identifies nonlinearities in modal space using the RFS method is discussed in [13]. Alternatively, a nonlinear modal identification approach based on the single nonlinear resonant mode concept $[14,15]$ and on a first-order frequency-domain approximation is proposed and applied in [16-19]. The forced frequency responses are expressed as a combination of a resonant nonlinear mode response and of linear contributions from the remaining modes. By a curve-fitting procedure, the amplitude-dependent nonlinear modal parameters may be identified from experimental responses close to the resonance.

In this paper, an attempt is made to extend experimental modal analysis to a practical nonlinear analog using the nonlinear normal mode (NNM) theory. NNMs offer a solid and rigorous mathematical tool for analyzing nonlinear oscillations, yet they have a clear conceptual relation to the classical linear normal modes (LNMs) [20,21]. Another appealing feature of NNMs is that they are capable of handling strong structural nonlinearity. Following the philosophy of force appropriation, the proposed method excites the NNMs of interest, one at a time. To this end, the phase lag quadrature criterion is generalized to nonlinear structures in order to locate single-NNM responses. Thanks to the invariance principle (i.e., if the motion is initiated on one specific NNM, the remaining NNMs remain quiescent for all time), the energy dependence of the NNM modal curves and their frequencies of oscillation can be extracted directly from experimental time data.

When used in conjunction with the numerical computation of the NNMs introduced in [22], the approach described herein leads to an integrated methodology for modal analysis of nonlinear vibrating structures (see Fig. 1). This methodology can, for instance, be used for validating dynamic models, as is routinely carried out for aerospace structures (e.g., ground vibration testing of aircrafts $[23,24]$ ).

The present paper is organized as follows. In the next section, the definition of an NNM is briefly recalled. The theoretical modal analysis consisting of the numerical NNM computation from a finite element model is also introduced. In Section 3, the proposed procedure for nonlinear EMA is then presented. The different concepts are illustrated using a nonlinear two-degree-of-freedom (2DOF) system. Finally in Section 4, the approach is demonstrated and assessed using numerical experiments carried out on a nonlinear beam.

\section{Theoretical modal analysis using nonlinear normal modes}

A detailed description of NNMs and of their fundamental properties (e.g., frequency-energy dependence, bifurcations and stability) is given in [20,21]. For completeness, the definition of an NNM is briefly reviewed in this section.

The free response of discrete conservative mechanical systems with $n$ degrees of freedom (DOFs) is considered, assuming that continuous systems (e.g., beams, shells or plates) have been spatially discretized using the finite element 
method. The general equations of motion are

$$
\mathbf{M} \ddot{\mathbf{x}}(t)+\mathbf{K} \mathbf{x}(t)+\mathbf{f}_{\mathrm{nl}}\{\mathbf{x}(t)\}=0
$$

where $\mathbf{M}$ is the mass matrix; $\mathbf{K}$ is the stiffness matrix; $\mathbf{x}, \dot{\mathbf{x}}$ and $\ddot{\mathbf{x}}$ are the displacement, velocity and acceleration vectors, respectively; $\mathbf{f}_{\mathrm{nl}}$ is the nonlinear restoring force vector, including stiffness terms only.

In the present study, as in [21], an extension of Rosenberg's definition [25-27] is considered. An NNM motion is therefore defined as a (non-necessarily synchronous) periodic motion of the undamped mechanical system (1). This NNM definition may appear restrictive in case of nonconservative systems. However, as shown in this paper and in [21], the damped dynamics can often be interpreted based on the topological structure of the NNMs of the underlying conservative system.

As evidenced in [22], this extended definition is particularly attractive when targeting a numerical computation of the NNMs. The approach followed here for the theoretical modal analysis consists in the numerical computation of undamped NNMs of nonlinear structures discretized by finite elements and governed by (1). The numerical method for the NNM computation relies on two main techniques, namely a shooting procedure and a method for the continuation of periodic solutions (i.e., NNM motions). A detailed description of the numerical algorithm is given in [22]. The NNMs are then obtained accurately, even in strongly nonlinear regimes, and in a fairly automatic manner.

One typical dynamical feature of nonlinear systems is the frequency-energy dependence of their oscillations. As a result, the modal curves and frequencies of NNMs depend on the total energy in the system. For illustration, a 2DOF system with a cubic stiffness is considered in this paper. The conservative system is depicted in Fig. 2, and its motion is governed by the equations

$$
\begin{gathered}
\ddot{x}_{1}+\left(2 x_{1}-x_{2}\right)+0.5 x_{1}^{3}=0 \\
\ddot{x}_{2}+\left(2 x_{2}-x_{1}\right)=0
\end{gathered}
$$

Fig. 3 shows the time series, the configuration space, the power spectral density (PSD) and two-dimensional projections of the phase space of three in-phase NNM motions of increasing energies. The NNM motion at low energy resembles that of the in-phase LNM of the underlying linear system. The modal curve (i.e., the NNM motion in the configuration space) is a straight line, there is one main harmonic component in the system response, and the motion in phase space is an ellipse. For the motion at moderate energy, the NNM is now a curve, and the presence of two harmonic components can be detected in the PSD. A clear departure from the LNM (harmonic) motion is observed. At high energy, this is even more enhanced. For instance, the motion in phase space is a strongly deformed ellipse. When moving from the low- to the high-energy NNM, the period of the motion decreases from 6.28 to $4.755 \mathrm{~s}$. This is due to the hardening characteristic of the cubic spring.

Due to frequency-energy dependence, the representation of NNMs in a frequency-energy plot (FEP) is particularly convenient. An NNM motion is represented by a point in the FEP, which is drawn at the fundamental frequency of the periodic motion and at the conserved total energy during the motion, which is the sum of the potential and kinetic energies. A branch, represented by a solid line, is a family of NNM motions possessing the same qualitative features.

For illustration, the conservative 2DOF system (2) is considered. The underlying linear system possesses two (in-phase and out-of-phase) LNMs. The FEP, computed using the numerical algorithm, is shown in Fig. 4. NNM motions in the configuration space (i.e., the modal curves) are inset. The backbone of the plot is formed by two branches, which represent in-phase $(S 11+)$ and out-of-phase (S $11-)$ synchronous NNMs. The indices in the notations are used to mention that the two masses vibrate with the same dominant frequency. These fundamental NNMs are the direct nonlinear extension of the corresponding LNMs. The FEP clearly shows that the nonlinear modal parameters, namely the modal curves and the corresponding frequencies of oscillation, have a strong dependence on the total energy in the system. The frequency of both the in-phase and out-of-phase NNMs increases with the energy level, which reveals the hardening characteristic of the cubic stiffness nonlinearity in the system.

Additional branches corresponding to internally resonant NNMs, as opposed to fundamental NNMs, bifurcate from the backbone at higher energy as evidenced in [21]. However, these modal interactions occurring through internal resonances are beyond the scope of the present study.

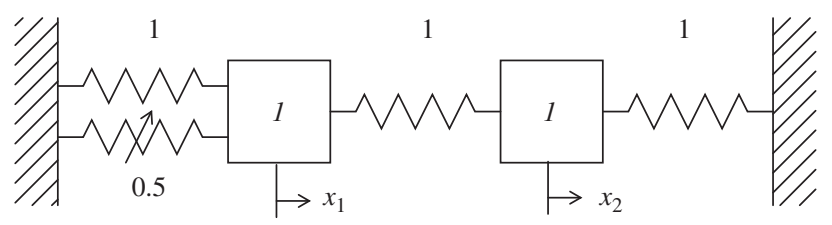

Fig. 2. Schematic representation of the 2DOF system example. 


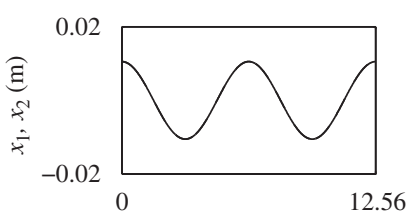

$t(\mathrm{~s})$
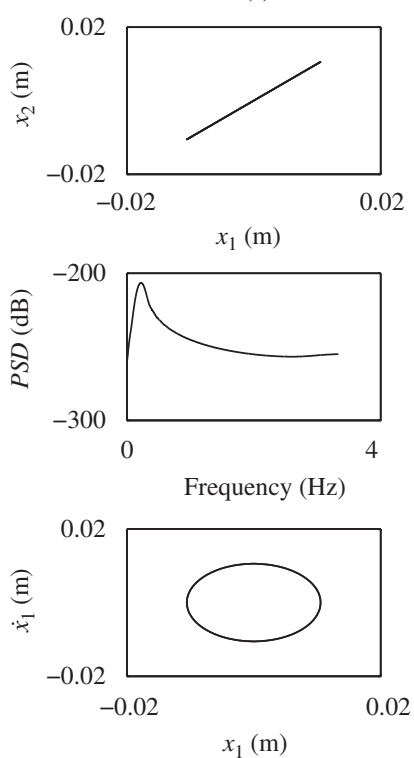

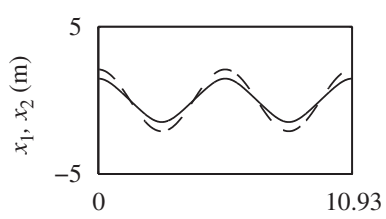

$t(\mathrm{~s})$
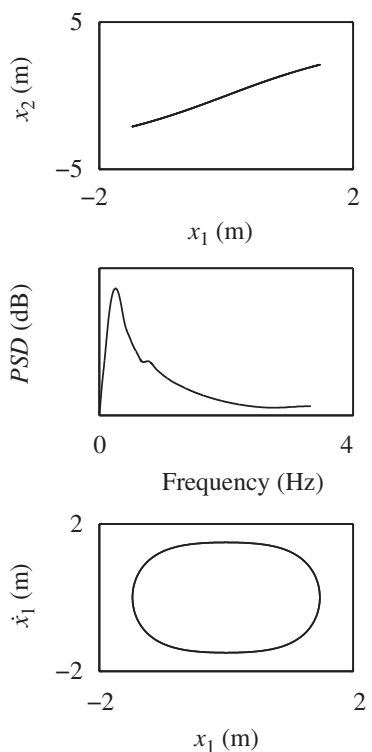

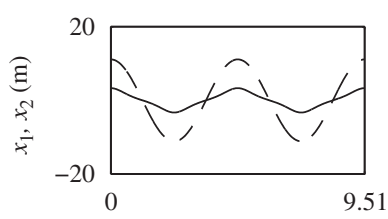

$t(\mathrm{~s})$
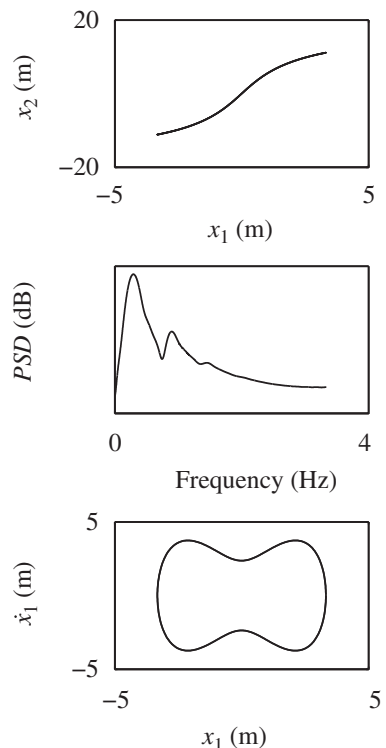

Fig. 3. Frequency-energy dependence of NNMs of the $2 \mathrm{DOF}$ system (2). From left to right: low- $\left(\left[x_{1}(0) x_{2}(0) \dot{x}_{1}(0) \dot{x}_{2}(0)\right]=[0.1050 .10500]\right)$,

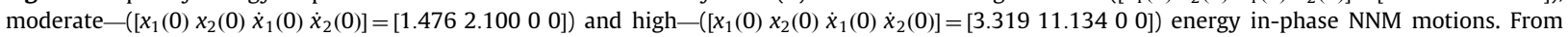
top to bottom: time series $\left(-: x_{1}(t) ;---: x_{2}(t)\right)$; configuration space; power spectral density of $x_{1}(t)$; two-dimensional projections of the phase space.

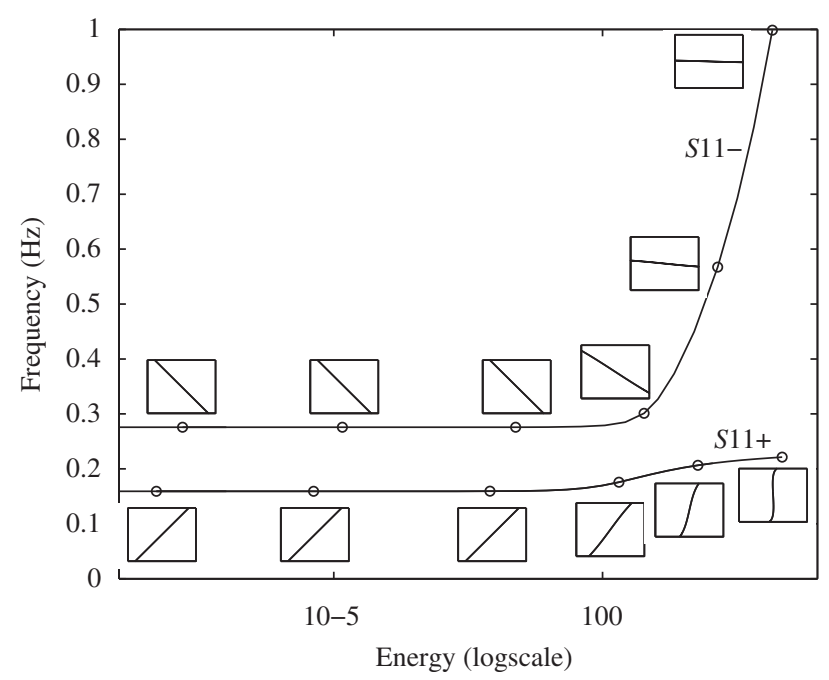

Fig. 4. Frequency-energy plot of the 2DOF system (2) computed with the numerical method. NNM motions depicted in the configuration space are inset. The horizontal and vertical axes in these plots are the displacements of the first and second DOFs, respectively.

\section{Experimental modal analysis using nonlinear normal modes}

There are two classical linear approaches for EMA. Phase separation methods excite several modes at once using either broadband excitation (e.g., hammer impact and random excitation) or swept-sine excitation covering the frequency range of interest. Phase resonance methods excite one mode at a time using multi-point sine excitation at the corresponding natural frequency [28]. A careful selection of the shaker locations is required to induce single-mode behavior. This process is also known as normal-mode tuning or force appropriation [29]. 
Phase resonance methods have been used for decades, particularly in the aerospace industry (e.g., for ground vibration testing of aircrafts [30] and modal survey of satellites [31,32]). They provide a very accurate identification of the modal parameters, but they are time-consuming. In order to reduce the testing time, phase separation methods are now used in conjunction with phase resonance methods [23,24]; these latter are reserved for modes that need a special attention (e.g., closely spaced modes and important modes for flutter computations).

\subsection{Proposed methodology}

In this paper, an attempt is made to extend EMA to a practical nonlinear analog using the NNM theory. Realizing that the general motion of a nonlinear system cannot be expressed as a superposition of NNMs, it is not clear how individual NNMs can be extracted using phase separation methods. This is why our focus is on the extension of phase resonance testing to nonlinear structures.

The present section introduces a two-step approach that extracts the NNM modal curves and their frequencies of oscillation directly from experimental data. This method is summarized in Fig. 5.

First, the method consists in exciting the system to induce single-NNM behavior at a specific energy level. To this end, an extension of force appropriation to nonlinear systems is developed. In particular, the phase lag of the response with respect to the applied force is used as an indicator to assess the quality of the appropriation. This first step, referred to as NNM force appropriation, is represented in the upper part of Fig. 5.

The second step turns off the excitation to track the energy dependence of the NNM of interest using the free damped response resulting from the appropriated NNM motion. A schematic representation is presented in the lower part of Fig. 5 . During this NNM free decay identification, the frequency-energy dependence may be extracted using time-frequency analysis. More details on the two steps of the procedure are given in the next sections.

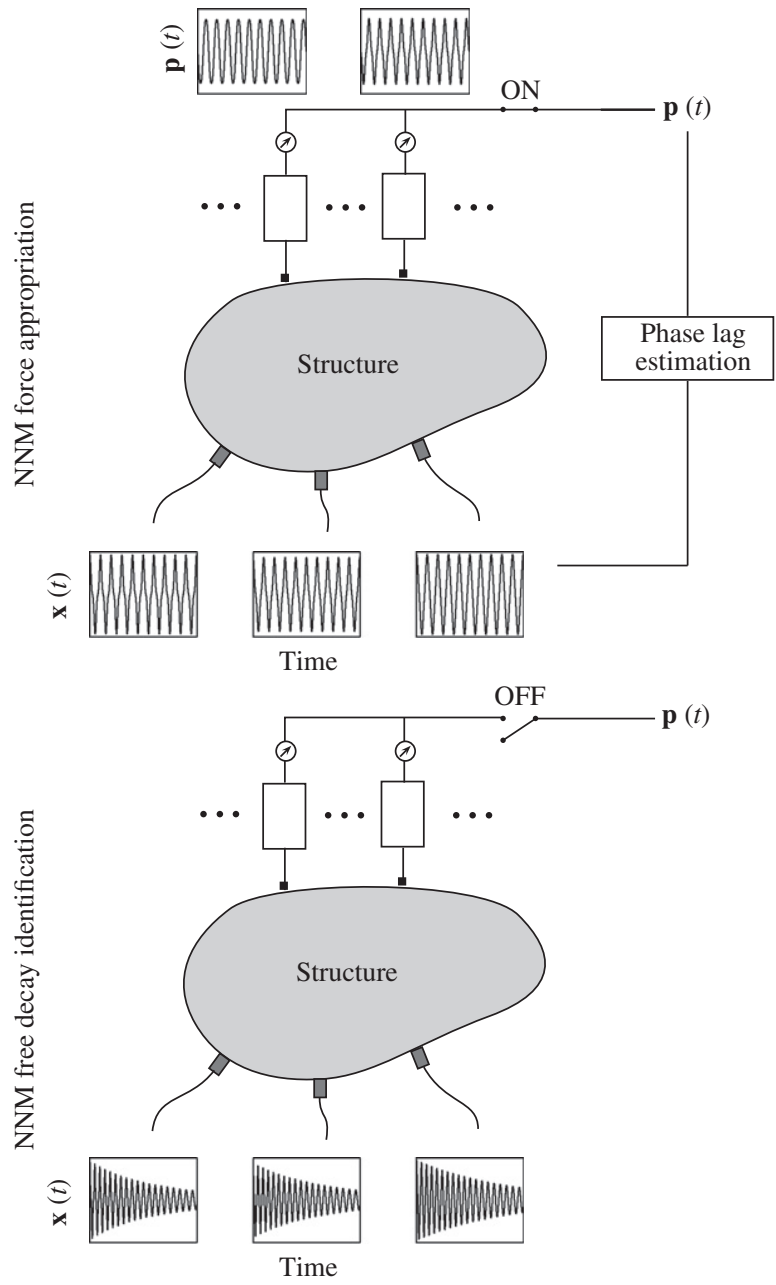

Fig. 5. Proposed methodology for experimental modal analysis of nonlinear systems. 
The methodology therefore consists in extracting one NNM at a time from the time series by means of this two-step procedure. In order to relate the forced and free responses of the damped system to the NNMs of the underlying conservative (i.e., undamped and unforced) system, the approach relies on three fundamental properties:

1. Forced responses of nonlinear systems at resonance occur in the neighborhood of NNMs [20].

2. According to the invariance property, motions that start out in the NNM manifold remain in it for all time [33].

3. For weak to moderate damping, its effect on the transient dynamics may be considered to be purely parasitic. The damped invariant manifold can therefore be approximated by the undamped invariant manifold. The free damped dynamics closely follows the NNM of the underlying undamped system [21,34,35].

From a practical viewpoint, the overall procedure for nonlinear EMA may be viewed as forced vibration testing where the appropriate force is applied as a burst excitation through several exciters for inducing single-NNM decay response. The philosophy of the resonant decay method [12] is then extended to the identification of NNMs of vibrating structures.

\subsection{NNM force appropriation}

For linear EMA, the force appropriation method generates appropriate harmonic forces at different locations on the structure to isolate a single normal mode. The measurement of the single-mode response then allows to extract the corresponding modal parameters.

Force appropriation is usually performed by adapting the frequency and the amplitude distribution of the multi-point excitation. According to the phase lag quadrature criterion (also called phase resonance criterion) [28,36], a linear damped structure vibrates according to one of the normal modes of the underlying conservative system if all degrees of freedom vibrate synchronously with a phase lag of $90^{\circ}$ with respect to the harmonic excitation. Hence, if the phase quadrature criterion is verified during the experimental testing, a single undamped normal mode is isolated, and the natural frequency and the mode shape can be identified.

The NNM framework is used herein to extend force appropriation to nonlinear structures in order to isolate and extract a single NNM at a time. To this end, the forced response of a nonlinear structure with linear viscous damping is considered

$$
\mathbf{M} \ddot{\mathbf{x}}(t)+\mathbf{C} \dot{\mathbf{x}}(t)+\mathbf{K} \mathbf{x}(t)+\mathbf{f}_{\mathrm{nl}}\{\mathbf{x}(t)\}=\mathbf{p}(t)
$$

where $\mathbf{p}(t)$ is the external excitation and $\mathbf{C}$ is the damping matrix. It is assumed that the nonlinear restoring force contains only stiffness nonlinearities.

In what follows, the necessary and sufficient conditions for isolating a single NNM are formulated. In this context, the excitation that induces a single NNM, termed appropriate excitation, is first examined in Section 3.2.1. A generalization of the phase lag quadrature criterion to nonlinear structures is next introduced in Section 3.2.2. It is important to note that when an 'NNM' is referred to in this paper, it stands for the NNM of the underlying conservative (no damping and no external force) system (1), i.e., the undamped NNM.

\subsubsection{Appropriate excitation}

Extracting a given NNM motion $\mathbf{x}_{\mathrm{nnm}}(t)$ of the underlying conservative system by means of appropriate excitation is equivalent to assume

$$
\mathbf{x}(t)=\mathbf{x}_{\mathrm{nnm}}(t)
$$

in the equations of motion of the forced and damped system (3). It leads to

$$
\mathbf{M} \ddot{\mathbf{x}}_{\mathrm{nnm}}(t)+\mathbf{C} \dot{\mathbf{x}}_{\mathrm{nnm}}(t)+\mathbf{K} \mathbf{x}_{\mathrm{nnm}}(t)+\mathbf{f}_{\mathrm{nl}}\left\{\mathbf{x}_{\mathrm{nnm}}(t)\right\}=\mathbf{p}_{\mathrm{nnm}}(t)
$$

where $\mathbf{p}_{\mathrm{nnm}}(t)$ is the corresponding appropriate excitation.

Because an undamped NNM motion $\mathbf{x}_{\mathrm{nnm}}(t)$ is defined as a periodic solution of the underlying conservative system, it follows that

$$
\mathbf{M} \ddot{\mathbf{x}}_{\mathrm{nnm}}(t)+\mathbf{K} \mathbf{x}_{\mathrm{nnm}}(t)+\mathbf{f}_{\mathrm{nl}}\left\{\mathbf{x}_{\mathrm{nnm}}(t)\right\}=\mathbf{0}
$$

Substituting expression (6) in Eq. (5), the excitation vector achieving a perfect appropriation (i.e., $\left.\mathbf{x}(t)=\mathbf{x}_{\mathrm{nnm}}(t)\right)$ of the damped system (3) is given by

$$
\mathbf{p}_{\mathrm{nnm}}(t)=\mathbf{C} \dot{\mathbf{x}}_{\mathrm{nnm}}(t)
$$

It expresses that the applied excitation has to compensate for the damping terms. This relationship shows that the appropriate excitation is periodic and has the same frequency components as the corresponding NNM motion (i.e., generally including multi-harmonic components).

An NNM motion is now expressed as a Fourier cosine series

$$
\mathbf{x}_{\mathrm{nnm}}(t)=\sum_{k=1}^{\infty} \mathbf{X}_{k}^{\mathrm{nnm}} \cos (k \omega t)
$$


where $\omega$ is the fundamental pulsation of the NNM motion and $\mathbf{X}_{k}$ is the amplitude vector of the $k$ th harmonic. In this paper, this type of motion is referred to as monophase NNM motion due to the fact that the displacements of all DOFs reach their extreme values simultaneously. Regarding the monophase NNM motion (8), the expression of the corresponding appropriate excitation is given by

$$
\mathbf{p}_{\mathrm{nnm}}(t)=-\sum_{k=1}^{\infty} \mathbf{C X}_{k}^{\mathrm{nnm}} k \omega \sin (k \omega t)
$$

Comparing Eqs. (8) and (9), the excitation of a monophase NNM is thus characterized by a phase lag of $90^{\circ}$ of each harmonic with respect to the displacement response.

For illustration, the 2DOF system represented in Fig. 2 is considered. Linear viscous damping is now taken into account to model dissipative effects in the structure. The forced response of the damped system is governed by the equations of motion

$$
\begin{gathered}
\ddot{x}_{1}+0.03 \dot{x}_{1}+\left(2 x_{1}-x_{2}\right)+0.5 x_{1}^{3}=p_{1} \\
\ddot{x}_{2}+0.01 \dot{x}_{2}+\left(2 x_{2}-x_{1}\right)=p_{2}
\end{gathered}
$$

which corresponds to moderate damping and where $p_{1}(t)$ and $p_{2}(t)$ represent the forces applied to this system.

Fig. 6 shows the time series and the configuration space of an undamped in-phase NNM motion at low energy (see point (a) in Fig. 14). This free motion of the underlying undamped system (2) resembles that of the in-phase LNM. The modal curve represented in the configuration space is a straight line. The appropriate excitation corresponding to this NNM motion calculated using expression (7) is depicted in Fig. 7. The steady-state forced response of the damped system (10) under this appropriate excitation is shown in Fig. 8. The response is in perfect agreement with the undamped and unforced NNM motion in Fig. 6. The Fourier coefficients of the forced response and of the appropriate excitation are represented in Fig. 9. There is one main harmonic component in the excitation and in the response: this fundamental frequency corresponds to the linear natural frequency of the in-phase LNM. A phase lag of $90^{\circ}$ is observed between the excitation and the response. At this low energy level, the nonlinear terms are negligible and the results are therefore consistent with the linear theory.

Similarly, an undamped in-phase NNM motion at high energy (see point (d) in Fig. 14) is considered. The time series and the configuration space of this NNM motion are depicted in Fig. 10. The related appropriate excitation is illustrated in Fig. 11. Fig. 12 shows that the corresponding steady-state forced response of the damped system (10) agrees very well with the undamped NNM motion of Fig. 10. The Fourier coefficients are presented in Fig. 13. For this strongly nonlinear regime, multi-harmonic components are observed. In addition to the fundamental frequency, higher harmonic terms are necessary to induce exactly this high-energy NNM motion. Fig. 13 also reveals the phase lag of $90^{\circ}$ of each harmonic of the excitation with respect to that of the response.

One important feature of the appropriate excitation is that it is energy-dependent. In particular, it is characterized by the same frequency-energy dependence as the corresponding NNM. As evidenced in (7), both signals possess the same frequency components. This is illustrated for the 2DOF example in Fig. 14 where the FEP of the in-phase NNM is depicted. The appropriate excitation and the resulting NNM motion are depicted for four different energy levels. Their Fourier coefficients are also represented. Clearly, when progressing from low to high energies, the fundamental frequency of the appropriate excitation increases, which is due to the hardening behavior of the system. In addition, multiple harmonics are necessary to induce single-NNM behavior.

(a)

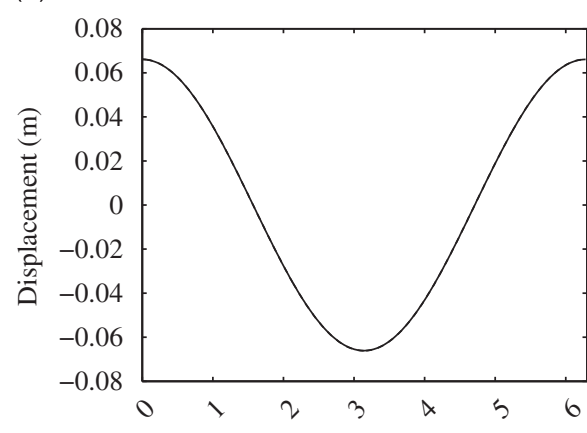

Time (s) (b)

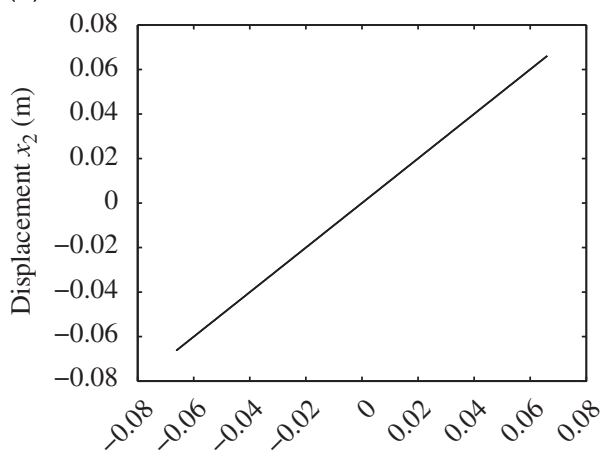

Displacement $x_{1}(\mathrm{~m})$

Fig. 6. Low-energy in-phase NNM motion of the conservative 2 DOF system (2). (a) Time series ( configuration space. 


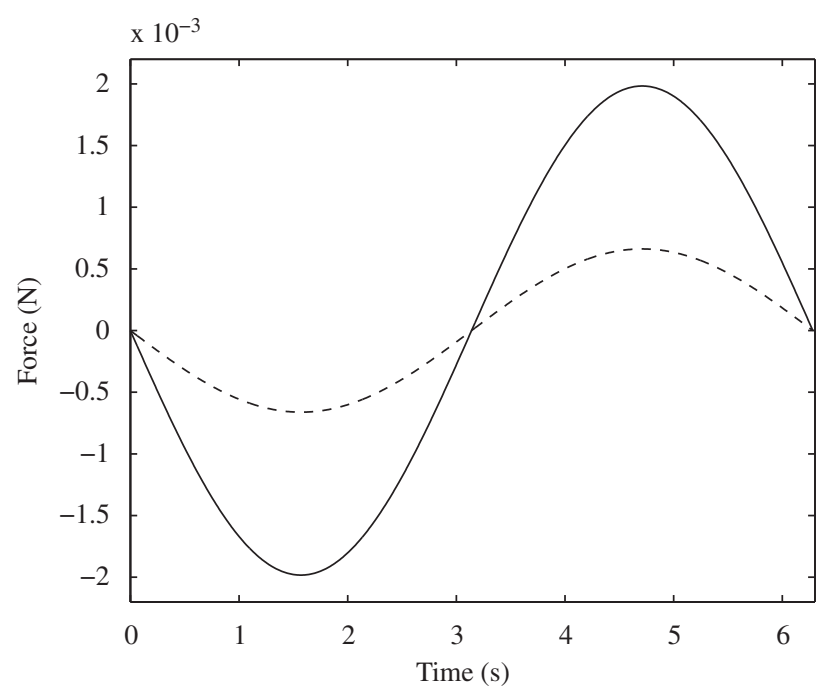

Fig. 7. Time series of the appropriate excitation corresponding to the low-energy NNM motion in Fig. $6\left(-\frac{-}{-} p_{1}(t) ;---: p_{2}(t)\right)$.

(a)

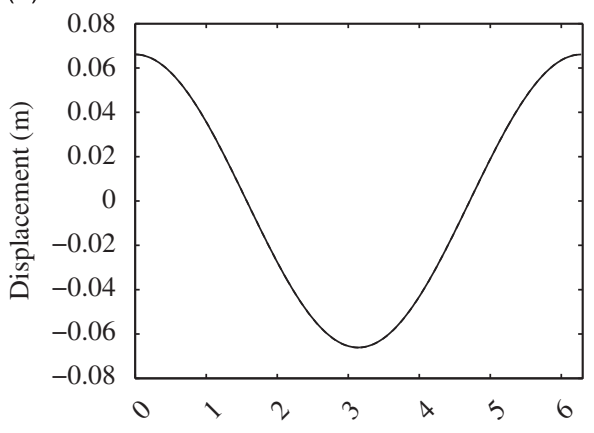

Time (s) (b)

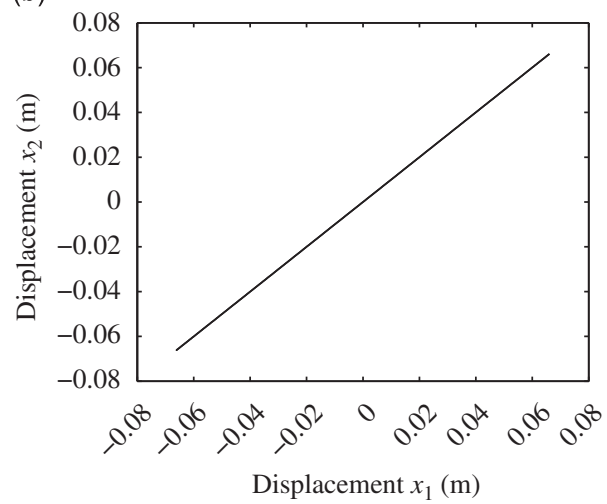

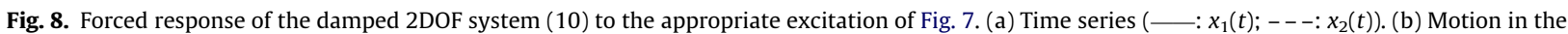
configuration space.

\subsubsection{Phase lag quadrature criterion for NNM force appropriation}

The previous section has demonstrated that nonlinear systems can successfully be forced according to a given NNM at a specific energy level through force appropriation. Specifically, if the applied force obeys Eq. (7), an NNM can be isolated. Because limited knowledge of the damping matrix is available in practical applications, a direct application of Eq. (7) is generally not possible, and one has to resort to an iterative force appropriation. In this context, an indicator highlighting that the NNM appropriation has effectively been achieved would be particularly useful. This is why the objective of the present section is to generalize the phase lag quadrature criterion (or phase resonance criterion) to nonlinear systems.

The forced response of the damped system (3) is examined when it vibrates according to a monophase periodic motion with a phase lag of $90^{\circ}$ of each harmonic with respect to the periodic excitation, i.e.,

$$
\mathbf{x}(t)=\sum_{k=1}^{\infty} \mathbf{x}_{k} \cos (k \omega t)
$$

and

$$
\mathbf{p}(t)=\sum_{k=1}^{\infty} \mathbf{P}_{k} \sin (k \omega t)
$$




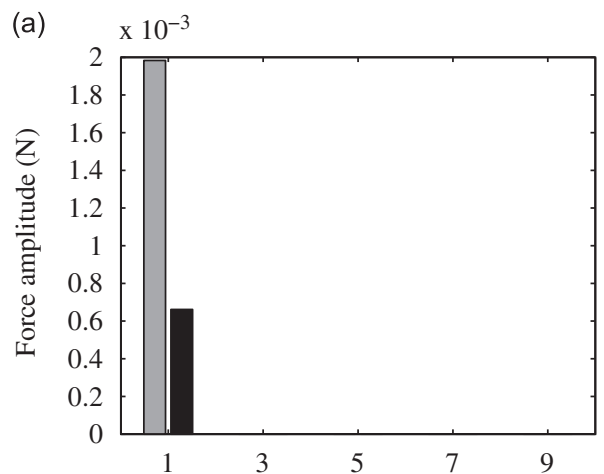

(b)
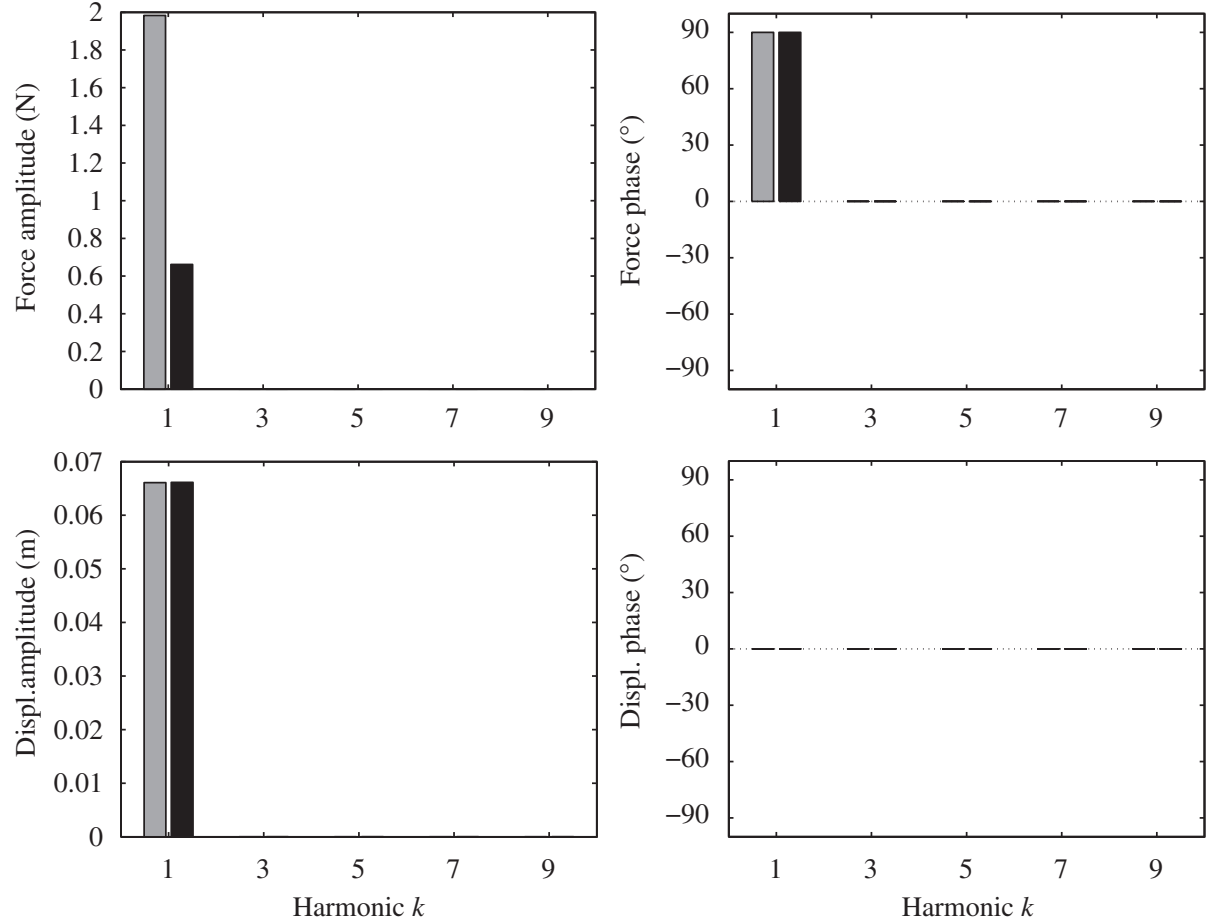

Fig. 9. Fourier coefficients of the appropriate excitation and the corresponding forced response of the damped 2DOF system (10) consisting of a low-energy NNM motion ((a) amplitude; (b) phase). Top plots: appropriate excitation (grey: $p_{1}$; black $p_{2}$ ); bottom plots: forced response (grey: $x_{1}$; black $x_{2}$ ).

(a)

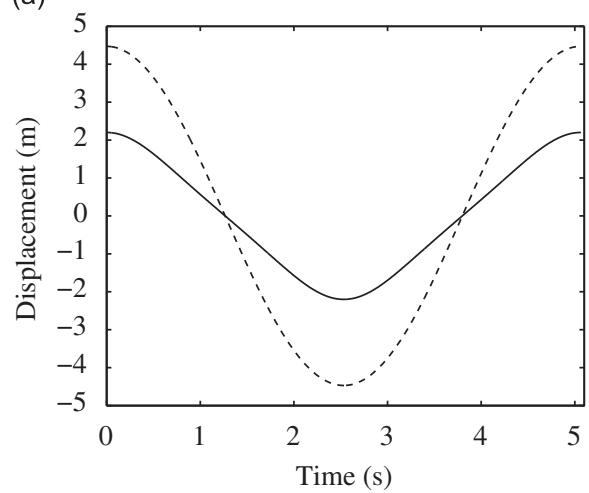

(b)

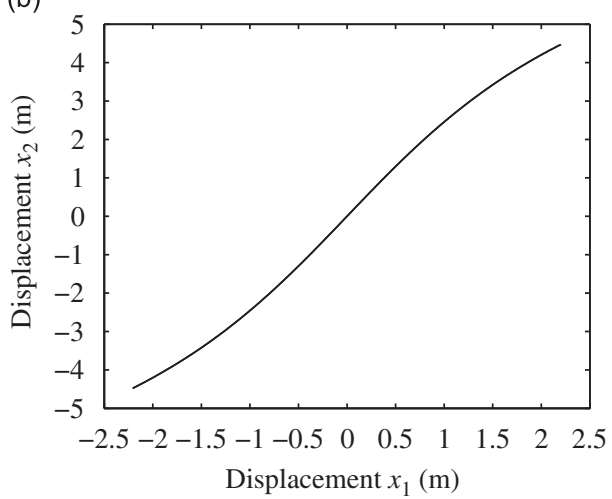

Fig. 10. High-energy in-phase NNM motion of the conservative 2DOF system (2). (a) Time series (- $x_{1}(t)$; $\left.---: x_{2}(t)\right)$. (b) Modal curve in the configuration space.

As the response is even with respect to the time, the nonlinear restoring forces can be written as a cosine series

$$
\mathbf{f}_{\mathrm{nl}}(\mathbf{x}(t))=\sum_{k=1}^{\infty} \mathbf{F}_{\mathrm{nl}, k} \cos (k \omega t)
$$

where $\mathbf{F}_{\mathrm{nl}, k}=\mathbf{F}_{\mathrm{n} 1, k}\left(\mathbf{X}_{r}\right)$ is a function of the harmonic coefficients $\mathbf{X}_{r}(r=1, \ldots, \infty)$.

In addition, the velocity and the acceleration vectors are computed by time differentiation

$$
\begin{gathered}
\dot{\mathbf{x}}(t)=-\sum_{k=1}^{\infty} k \omega \mathbf{X}_{k} \sin (k \omega t) \\
\ddot{\mathbf{x}}(t)=-\sum_{k=1}^{\infty} k^{2} \omega^{2} \mathbf{X}_{k} \cos (k \omega t)
\end{gathered}
$$




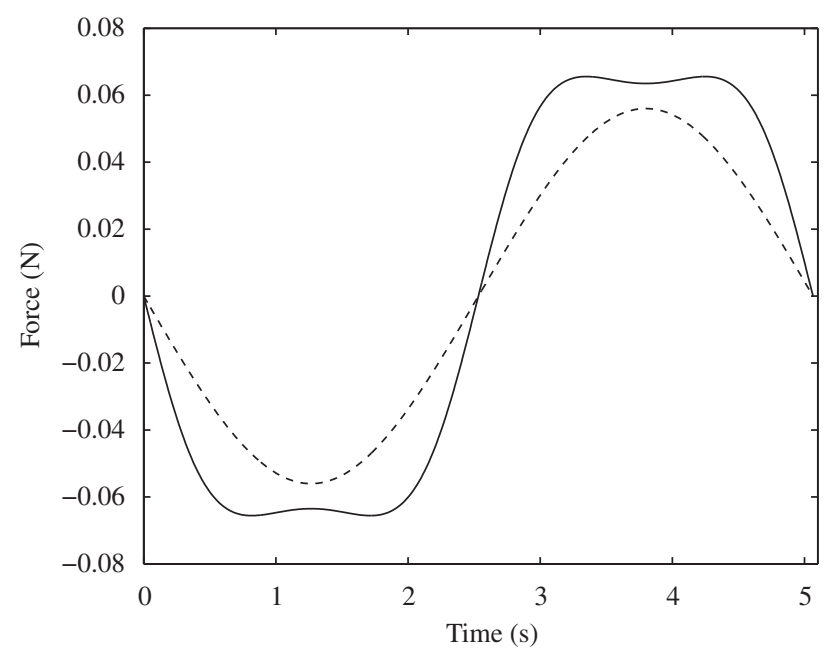

Fig. 11. Time series of the appropriate excitation corresponding to the high-energy NNM motion in Fig. $10\left(-: p_{1}(t) ;---: p_{2}(t)\right)$.

(a)

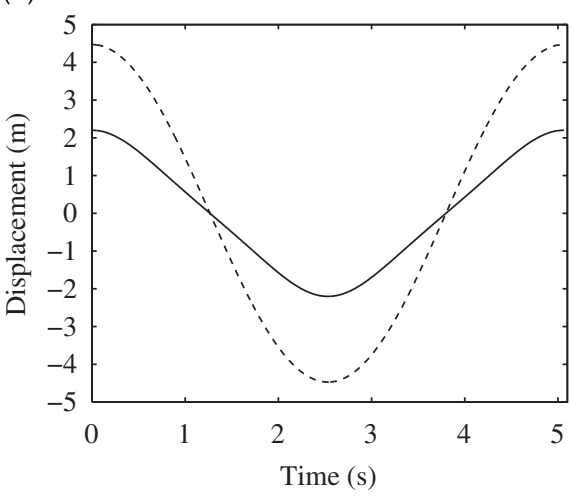

(b)

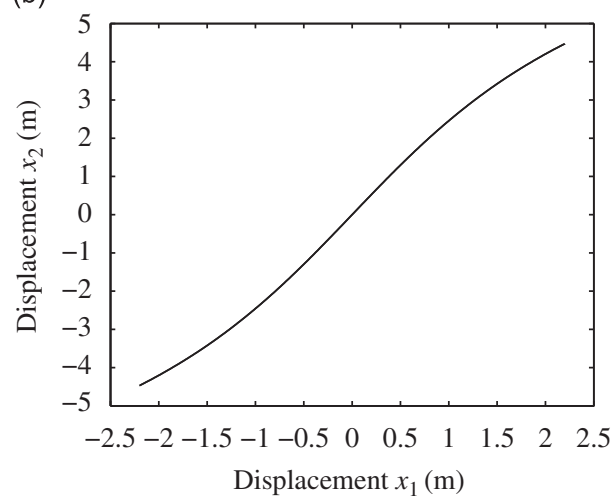

Fig. 12. Forced response of the damped 2DOF system (10) to the appropriate excitation of Fig. 11. (a) Time series (the configuration space.

By inserting all these expressions into the equations of motion (3), we obtain

$$
-\mathbf{M} \sum_{k=1}^{\infty} k^{2} \omega^{2} \mathbf{X}_{k} \cos (k \omega t)-\mathbf{C} \sum_{k=1}^{\infty} k \omega \mathbf{X}_{k} \sin (k \omega t)+\mathbf{K} \sum_{k=1}^{\infty} \mathbf{X}_{k} \cos (k \omega t)+\sum_{k=1}^{\infty} \mathbf{F}_{\mathrm{n}, k} \cos (k \omega t)=\sum_{k=1}^{\infty} \mathbf{P}_{k} \sin (k \omega t)
$$

By balancing the coefficients of respective harmonics, it follows that

$$
\begin{gathered}
-k^{2} \omega^{2} \mathbf{M} \mathbf{X}_{k}+\mathbf{K} \mathbf{X}_{k}+\mathbf{F}_{\mathrm{n} 1, k}\left(\mathbf{X}_{r}\right)=\mathbf{0} \\
-k \omega \mathbf{C} \mathbf{X}_{k}=\mathbf{P}_{k}
\end{gathered}
$$

for $k=1, \ldots, \infty$. So, the external force is given by

$$
\mathbf{p}(t)=\sum_{k=1}^{\infty} \mathbf{P}_{k} \sin (k \omega t)=-\sum_{k=1}^{\infty} k \omega \mathbf{C X}_{k} \sin (k \omega t)=\mathbf{C} \dot{\mathbf{x}}(t)
$$

and the periodic response $\mathbf{x}(t)$ is a solution of the underlying conservative system (1). The response $\mathbf{x}(t)$ is therefore an undamped NNM motion of the system: the NNM force appropriation is realized.

In conclusion, the phase lag quadrature criterion, valid for linear systems, can be generalized to monophase NNM motions of nonlinear structures, where the phase lag is defined with respect to each harmonic of the monophase signals.

In other words, if the response (in terms of displacements or accelerations) across the structure is a monophase periodic motion in quadrature with the excitation, the structure vibrates according to a single NNM of the underlying conservative 
(a)
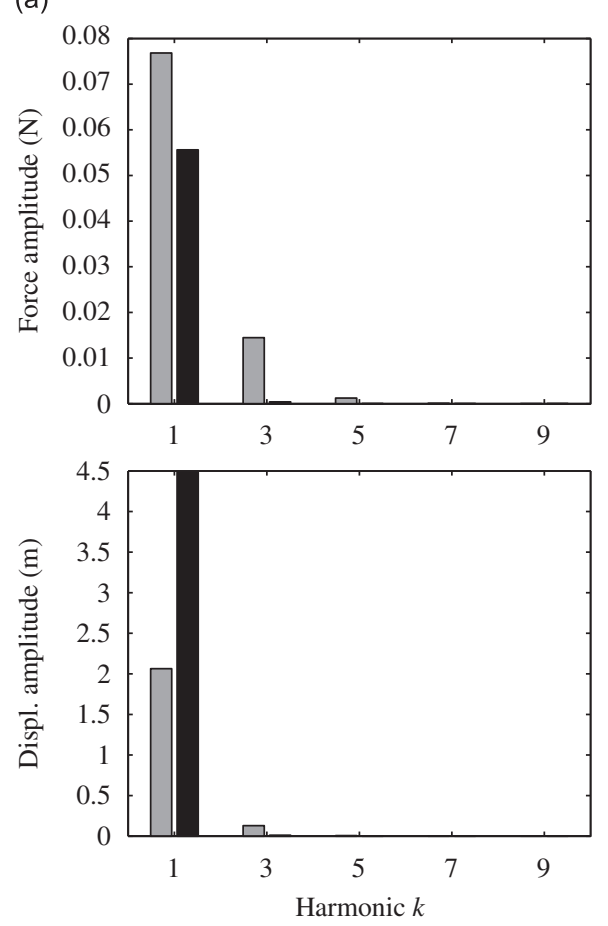

(b)
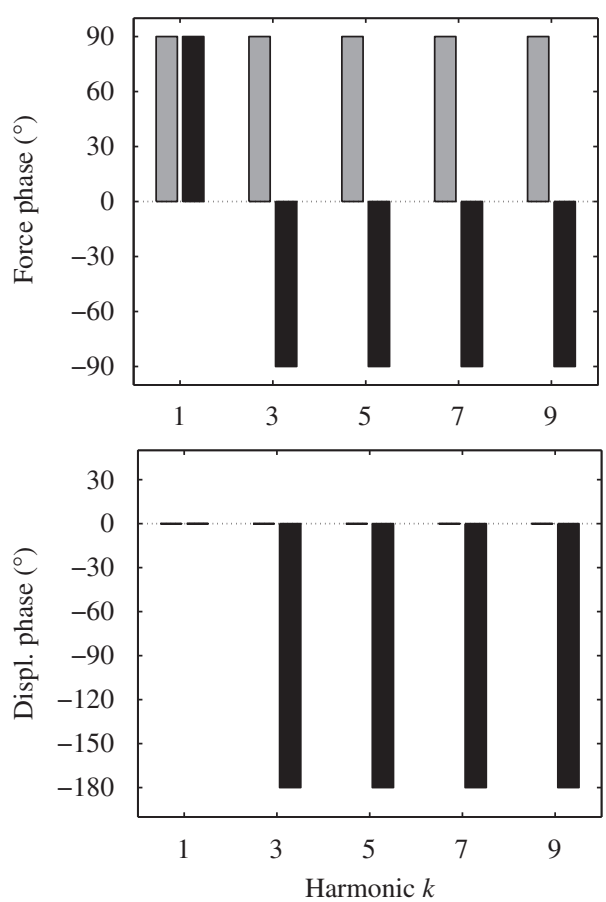

Fig. 13. Fourier coefficients of the appropriate excitation and the corresponding forced response of the damped $2 \mathrm{DOF}$ system (10) consisting of a high-energy NNM motion ((a) amplitude; (b) phase). Top plots: appropriate excitation (grey: $p_{1}$; black $p_{2}$ ); bottom plots: forced response (grey: $x_{1}$; black $x_{2}$ ).

system. The phase lag of the generated monophase excitation with respect to the response can thus be used as an indicator of the NNM appropriation.

No direct constructive method exists to determine the appropriate excitation of a given NNM. Such an excitation has to be derived through successive approximations based on this indicator. For nonlinear structures, in addition to the spatial distribution of the multi-point excitation, the amplitude distribution of harmonic terms has also to be tuned. In fact, the frequency-energy dependence of nonlinear systems prevents the direct separation of space and time in the governing equations of motion, which may complicate the experimental realization of force appropriation from a theoretical viewpoint.

The phase resonance feature has been highlighted in the previous section. For NNM motions of the damped 2DOF system, a phase lag of $90^{\circ}$ of the displacement response with respect to the monophase appropriate excitation has been shown (see Figs. 7 and 11). The phase lag quadrature criterion is now used for estimating the quality of the NNM appropriation. The periodic forced responses of the damped 2DOF system (10) to a harmonic force of frequency $\omega$ and amplitude $F$ applied to the first DOF

$$
\begin{gathered}
\ddot{x}_{1}+0.03 \dot{x}_{1}+\left(2 x_{1}-x_{2}\right)+0.5 x_{1}^{3}=F \sin (\omega t) \\
\ddot{x}_{2}+0.01 \dot{x}_{2}+\left(2 x_{2}-x_{1}\right)=0
\end{gathered}
$$

are analyzed. An imperfect force appropriation (i.e., $p_{2}(t)=0$ and purely harmonic excitation) is purposely considered to investigate the robustness of the proposed procedure.

The nonlinear frequency responses close to the resonance of the in-phase mode were computed using shooting and continuation methods and are depicted in Fig. 15. They are given in terms of amplitude and phase lag (of the fundamental frequency component) of the displacement response for increasing forcing amplitudes. It is observed that the phase quadrature criterion is almost verified close to the forced resonance. For $F=0.2 \mathrm{~N}$, i.e., at the point marked by a square, the phase lag is equal to $90^{\circ}$ and $91^{\circ}$ for the first and second DOFs, respectively. Fig. 16 represents the time series of the displacement response for $F=0.2 \mathrm{~N}$. Clearly, the displacement is practically monophase with a phase lag around $90^{\circ}$ with respect to the excitation $p_{1}(t)=F \sin (\omega t)$.

These results also confirm that forced responses of nonlinear systems at resonance occur in the neighborhood of NNMs. The backbone of the in-phase undamped NNM (represented in Fig. 4) is expressed in terms of amplitude and is displayed using a dashed line in Fig. 15. This backbone curve traces the locus of the frequency response peaks. In addition, the undamped NNM motion corresponding to the same frequency as the point marked by a square in Fig. 15 is shown in 


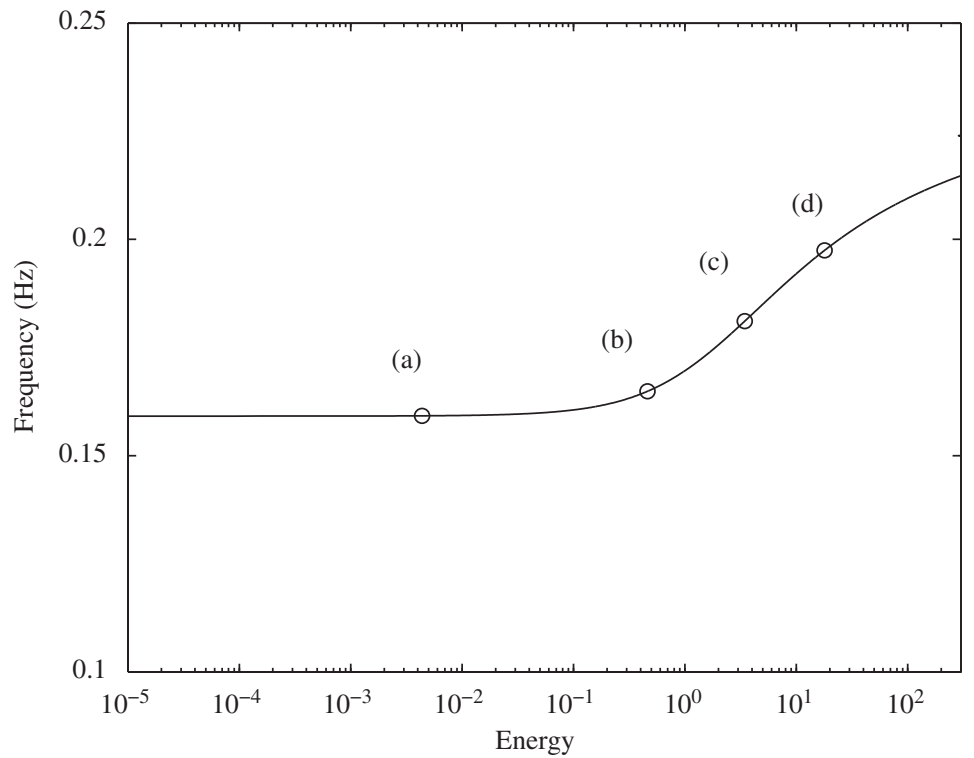

(a)
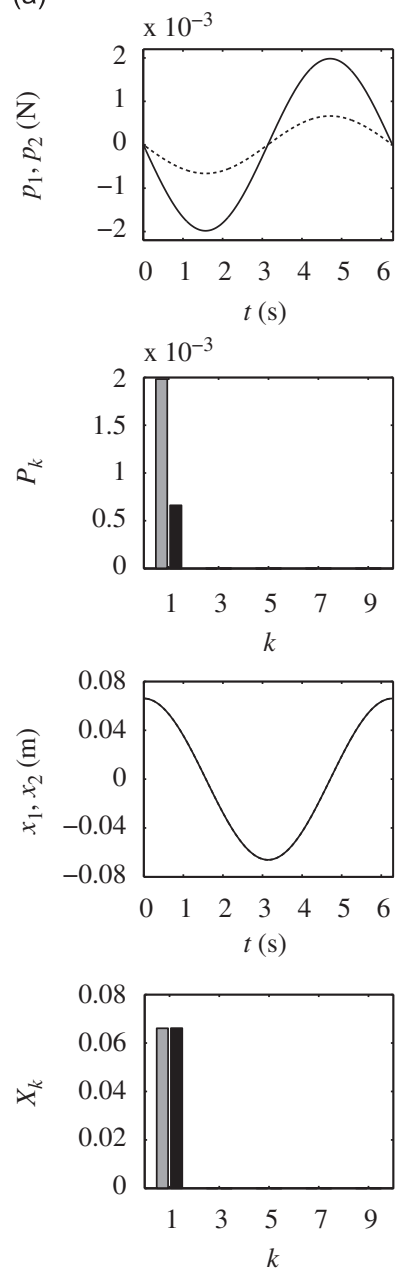

(b)
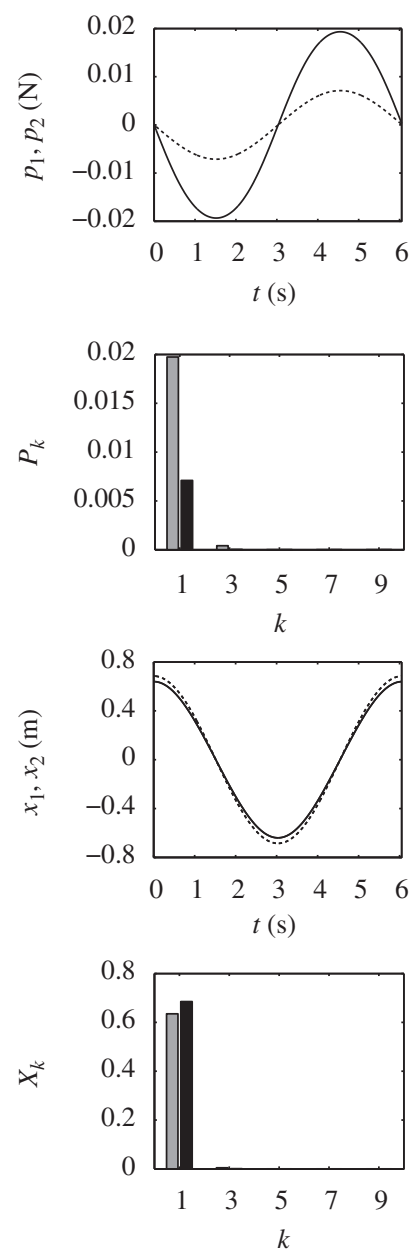

(c)
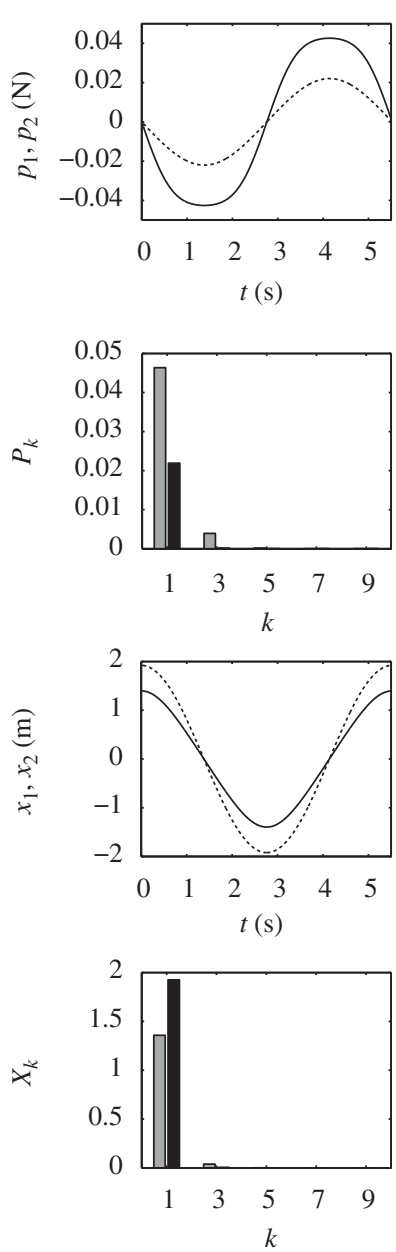

(d)
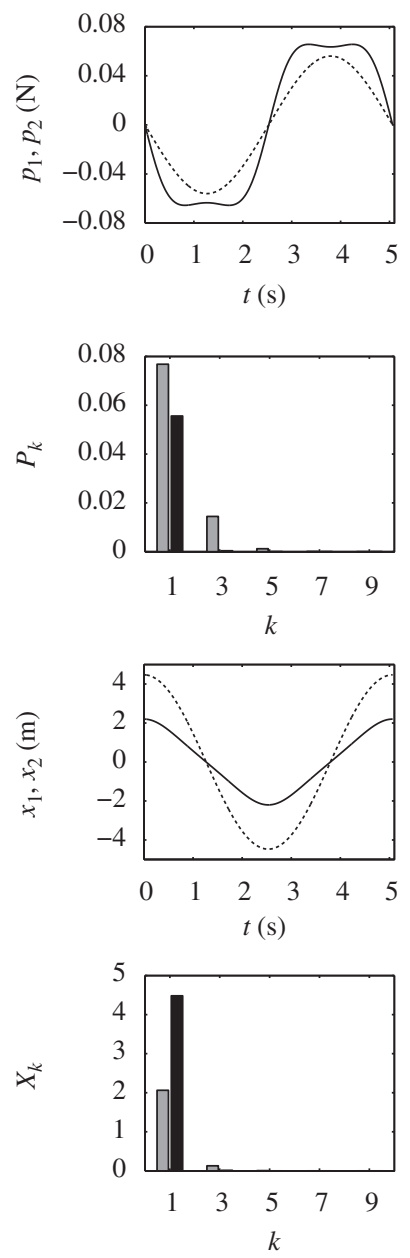

Fig. 14. Frequency-energy dependence of the appropriate excitation for the 2DOF system (10). Top plot: FEP of the in-phase NNM of the underlying undamped system (2). Bottom plots (from top to bottom): time series of the appropriate excitation $\left(-: p_{1}(t) ;---: p_{2}(t)\right.$ ); Fourier coefficients of the appropriate excitation (grey: $p_{1}$; black: $\left.p_{2}\right)$; time series of the corresponding NNM motion $\left(-: x_{1}(t)\right.$; - - - : $x_{2}(t)$ ); Fourier coefficients of the NNM motion (grey: $x_{1}$; black: $x_{2}$ ). 
(a)
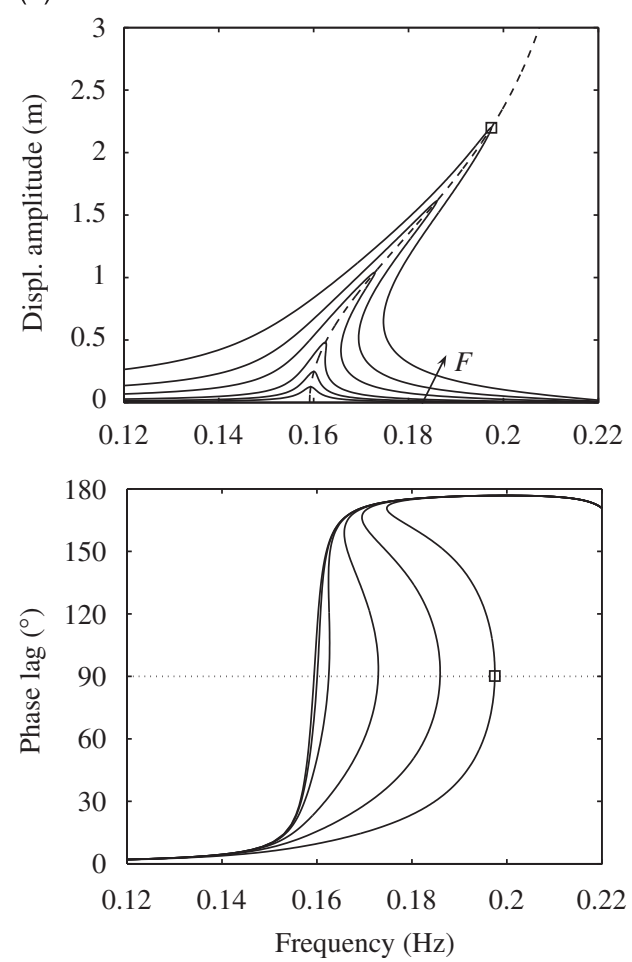

(b)
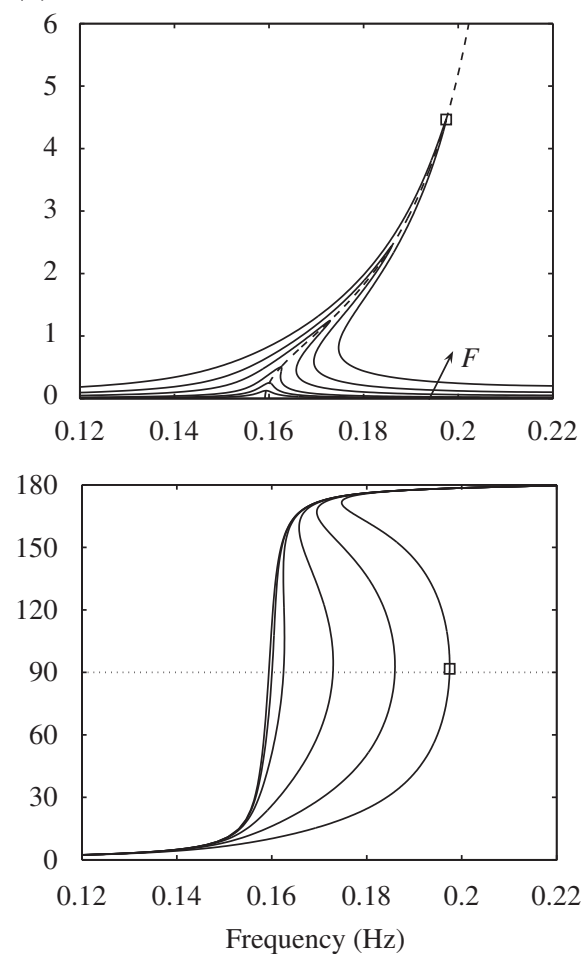

Fig. 15. Nonlinear frequency responses of the damped 2DOF system (18) close to the first resonant frequency (six different forcing amplitudes $F$ : 0.005 , $0.01,0.02,0.05,0.1,0.2 \mathrm{~N}$ ). The dashed line is the backbone of the first undamped NNM (computed by means of the numerical algorithm). Top plots: displacement amplitude. Bottom plots: phase lag of the displacement with respect to the excitation. (a) $x_{1}$; (b) $x_{2}$.

(a)

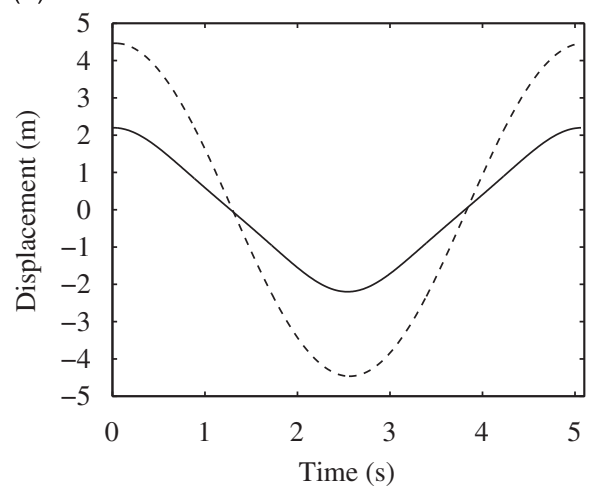

(b)

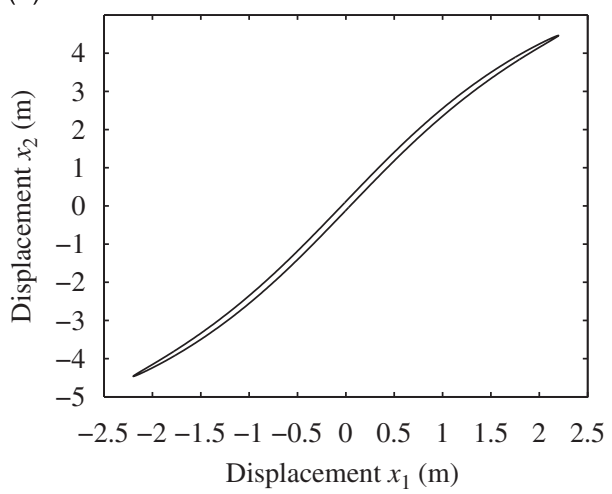

Fig. 16. Forced response (corresponding to $F=0.2 \mathrm{~N}$ and marked by a square in Fig. 15) of the damped 2DOF system (18). (a) Time series (—- $x_{1}(t) ;---: x_{2}(t)$ ). (b) Motion in the configuration space.

Fig. 10. An excellent agreement is observed between the time series of Figs. 10 and 16, the mean-square error (MSE) being around 0.01 percent.

It is interesting that no forcing on the second DOF and no higher harmonic terms were necessary to isolate this highenergy NNM motion. This is an appealing feature for future practical realizations, at least for structures with relatively well-separated modes. A constructive method for inducing single-NNM behavior could be to perform successive adjustments of a stepped sine excitation until the phase lag criterion is verified. This approach is considered in Section 4 where the proposed methodology is demonstrated using simulated experiments of a nonlinear beam.

It is worth pointing out that the phase lag quadrature criterion, demonstrated here for linear viscous damping, still holds in case of nonlinear damping provided that the damping force $\mathbf{f}_{d, \mathrm{nl}}$ is an odd function of the velocity only, i.e., $\mathbf{f}_{d, \mathrm{nl}}(\dot{\mathbf{x}})=-\mathbf{f}_{d, \mathrm{nl}}(-\dot{\mathbf{x}})$. For monophase motion (11), the damping terms are indeed expressed as a sine series and therefore have to be compensated by the applied force (12). However, if the stiffness and damping nonlinearities are coupled 
(i.e., $\mathbf{f}_{\mathrm{nl}}=\mathbf{f}_{\mathrm{nl}}(\mathbf{x}, \dot{\mathbf{x}})$ ), this criterion generally disappears at the same time as the concept of NNMs of the underlying conservative system.

\subsection{NNM free decay identification}

By means of nonlinear force appropriation, the forced response of the damped system (3) may be restricted to a single undamped NNM at a specific energy level. In view of the frequency-energy dependence, successive appropriate excitations at different force levels have to be considered to provide a complete characterization of the NNM of interest. This may complicate the experimental realization.

The alternative strategy proposed here consists in exploiting the NNM invariance property. When a high-energy NNM motion is isolated using force appropriation, the excitation is stopped to obtain the resulting free damped response. Due to invariance, this free decay response initiated on the undamped NNM remains close to it when energy decreases. Using this procedure, the energy dependence of the NNM modal curves and the corresponding frequencies of oscillation may easily be extracted from the single-mode free damped response at each measurement location.

As mentioned above, according to invariance, the resulting free damped response closely follows the corresponding undamped NNM provided that the damping is moderate [21]. The relation between the two responses is only phenomenological, nevertheless it enables one to interpret the damped response in terms of NNM motions of the underlying undamped system. In fact, the damped manifold corresponds to the exact invariant manifold of the damped dynamics. However, for lightly damped structures, the latter may be approximated by the undamped NNM that can be viewed as an attractor of the free damped response. For illustration, the 2DOF system (10) is considered. From the appropriated in-phase undamped NNM motion of Fig. 12, the resulting free damped response when the excitation is removed is depicted in Fig. 17. Fig. 18 compares the manifold corresponding to the in-phase undamped NNM with the free

(a)

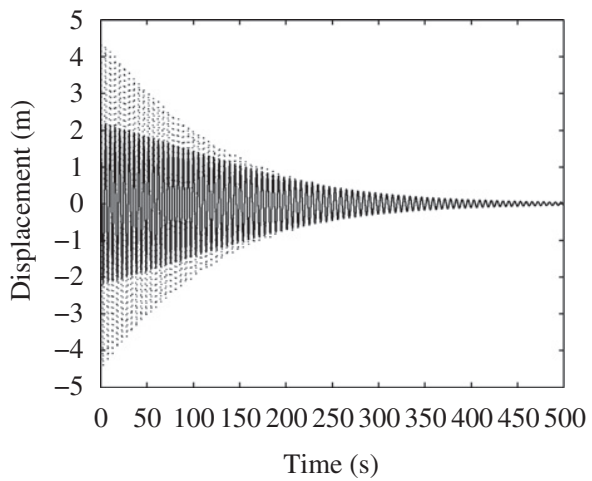

(b)

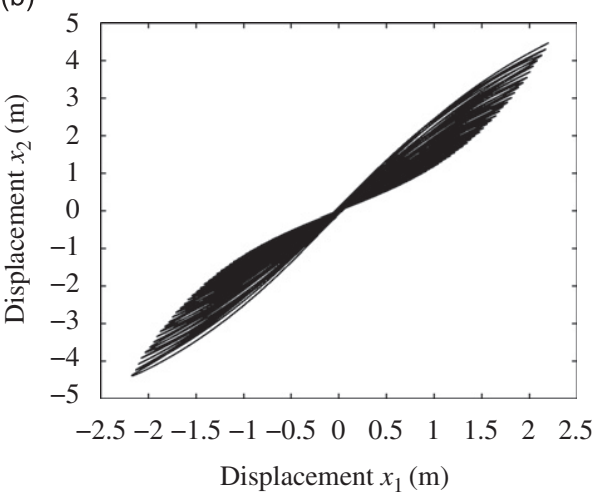

Fig. 17. Free response of the damped 2DOF system (10) initiated from the in-phase NNM motion of Fig. 12. (a) Time series $\left(-: x_{1}(t) ;---: x_{2}(t)\right)$. (b) Motion in the configuration space.

(a)

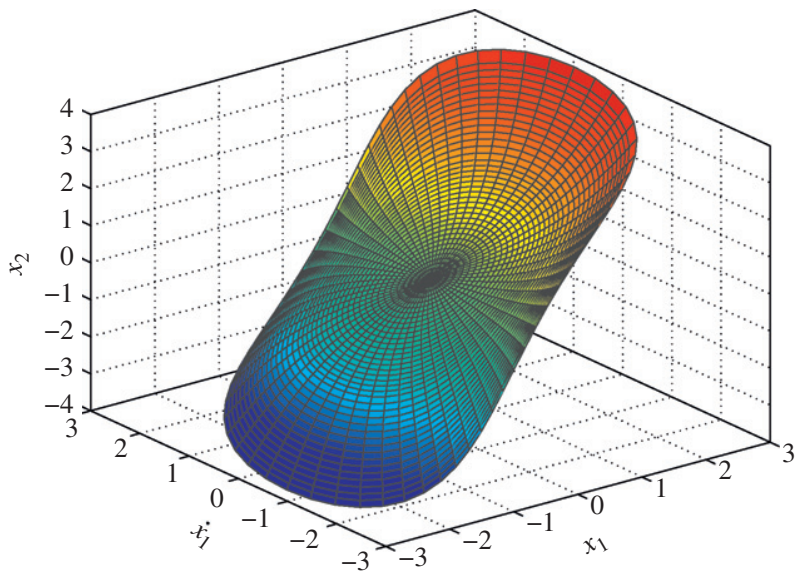

(b)

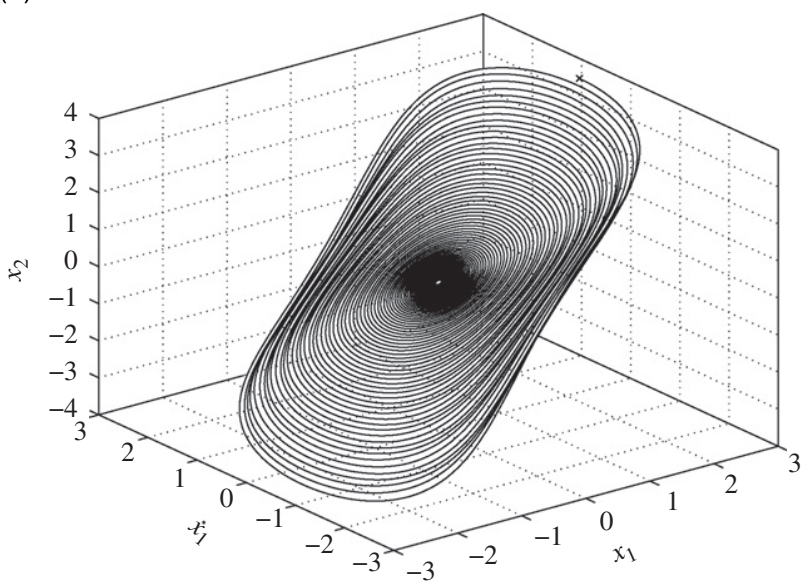

Fig. 18. Invariant manifold of the in-phase NNM of the 2DOF system. (a) NNM manifold of the underlying undamped system (2). (b) Free response of the damped system (10). 
damped response represented in the same projection of phase space. Clearly, it confirms that the free damped response traces the NNM manifold of the underlying undamped system with very good accuracy when energy decreases.

NNM extraction: As a result, the NNM modal curves may be extracted directly from the single-NNM free decay response. They are obtained by representing the time series in the configuration space for one oscillation around specific time instants, associated with different energy levels. It is illustrated in Fig. 19 for the 2DOF system around four distinct instants.

To compute the oscillation frequency of NNMs, time-frequency analysis is considered. Time-frequency analysis is a versatile tool for analyzing nonstationary signals; i.e., signals whose spectral contents vary with time. It has been successfully exploited in structural dynamics, e.g., for linear and nonlinear system identification [37-39]. The continuous wavelet transform (CWT) [40] is used in this paper. In contrast to the Fourier transform, which assumes signal stationarity, the CWT involves a windowing technique with variable-sized regions. Small time intervals are considered for highfrequency components, whereas the size of the interval is increased for lower-frequency components. The CWT can therefore track the temporal evolution of the instantaneous frequencies, which makes it an effective tool for analyzing

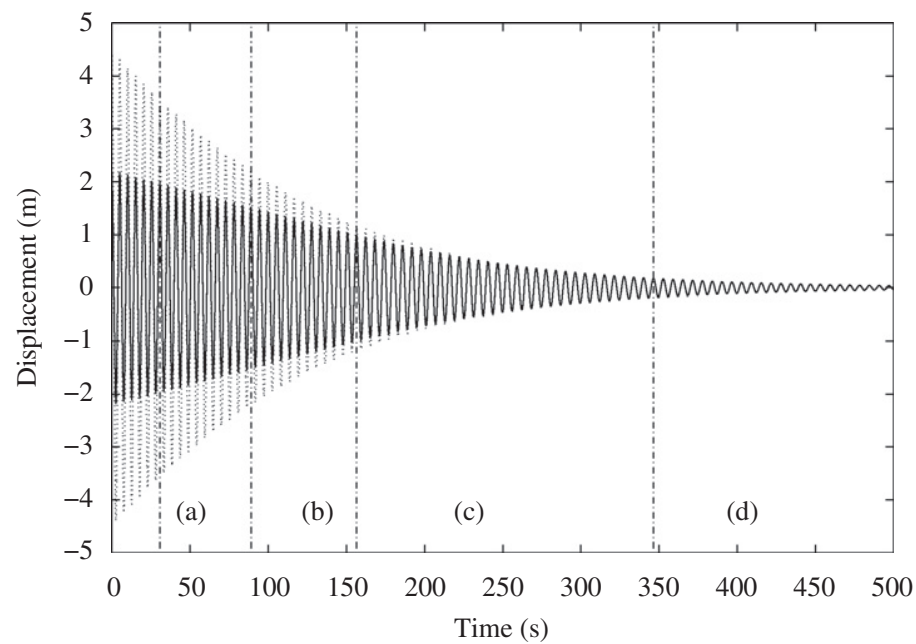

(a)

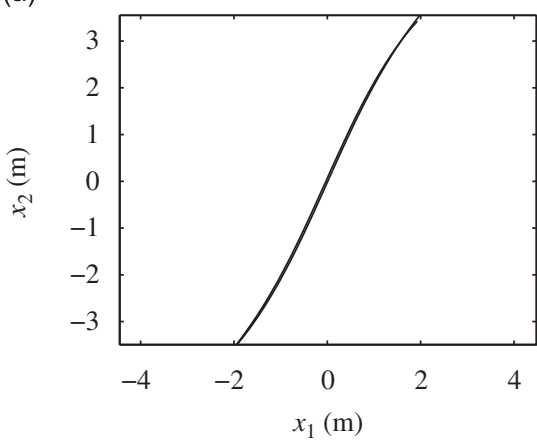

(c)

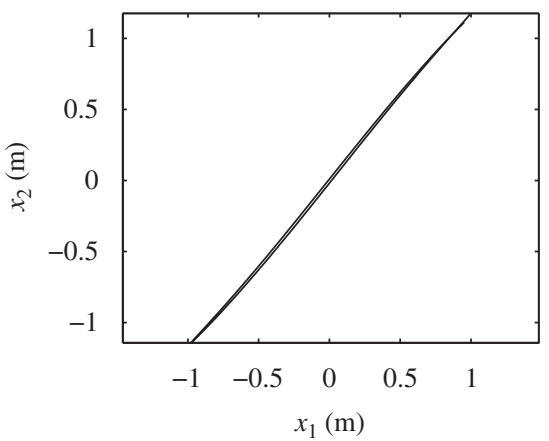

(b)

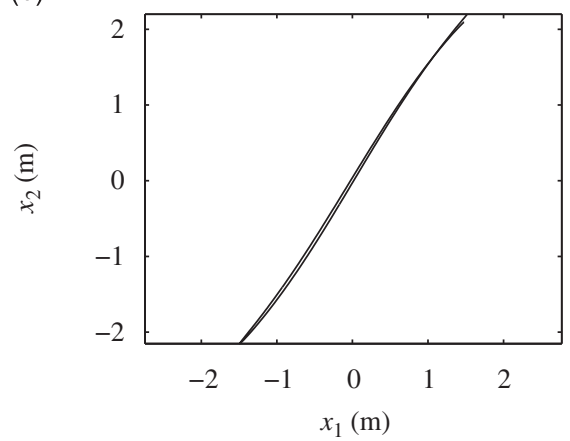

(d)

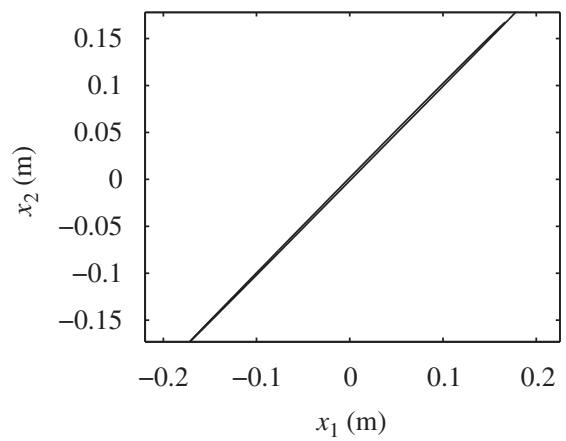

Fig. 19. Extraction of NNM modal curves during the free decay. Top plot: time series of the resulting single-NNM free response of the damped $2 \mathrm{DOF}$ system (10). Bottom plots: NNM modal curves extracted from the time series around four time instants marked in the top plot by (a), (b), (c) and (d). 
nonlinear signals. Using the CWT, the oscillation frequency of the NNM may then be extracted from the time series of the free damped response. The usual representation of the transform is to plot its modulus as a function of time and frequency in a three-dimensional or contour plot. For illustration, the CWT of the free decay response of the 2DOF system represented in Fig. 17 is shown in Fig. 20. The instantaneous frequency decreases with time, and hence with energy, which reveals the hardening characteristic of the system.

Reconstructed FEP: When the total energy (i.e., the sum of the kinetic and potential energies) in the system can be determined, the experimental FEP can be reconstructed in a straightforward manner by substituting the instantaneous energy in the system for time: (i) the backbone expressing the frequency-energy dependence of the NNM is provided by the CWT, (ii) the obtained modal curves around different energy levels are superposed in the plot.

For the 2DOF example, the experimental FEP calculated from the time series of the free damped response is represented in Fig. 21. It displays the experimental backbone determined through the CWT and the experimental modal curves. For comparison, the theoretical FEP of the undamped NNM computed in Section 2 from the equations of motion is also illustrated. Except the CWT edge effects, a perfect agreement is obtained between the two FEPs, which shows again that the undamped NNM is an attractor for the damped trajectories. In the present case, the linear modal damping ratios are 1 and 0.6 percent, but we note that this result holds for higher damping ratios.

In summary, it is thus validated that the free damped dynamics can be interpreted based on the topological structure of the NNM of the underlying conservative system. As a result, one can fully reconstruct the FEP and extract the modal curves together with the oscillation frequencies of the NNM using the proposed procedure.

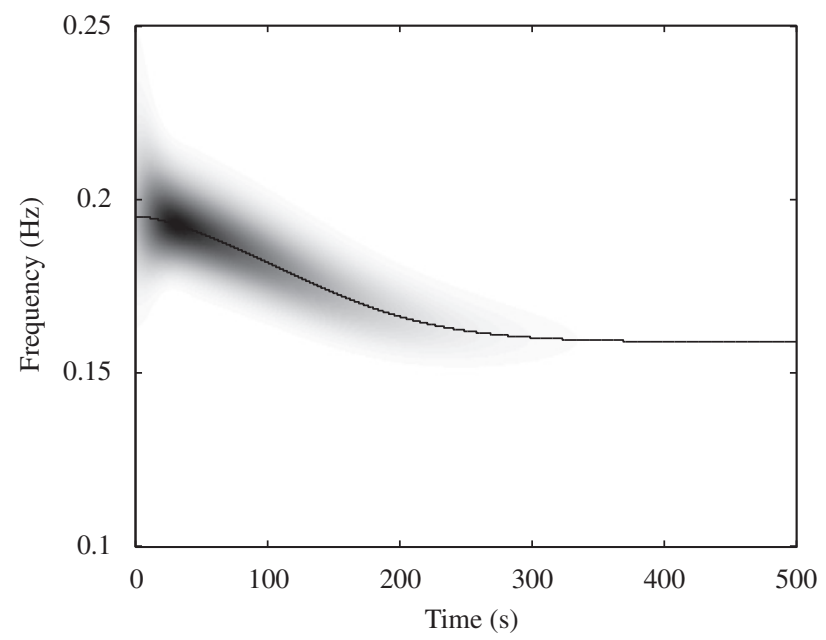

Fig. 20. Extraction of oscillation frequencies of the NNM during the free decay. Temporal evolution of the instantaneous frequency of the single-NNM free response of the damped 2DOF system (10) computed using the CWT. The solid line is the ridge of the transform; i.e., the locus of the maxima at each time instant.

(a)

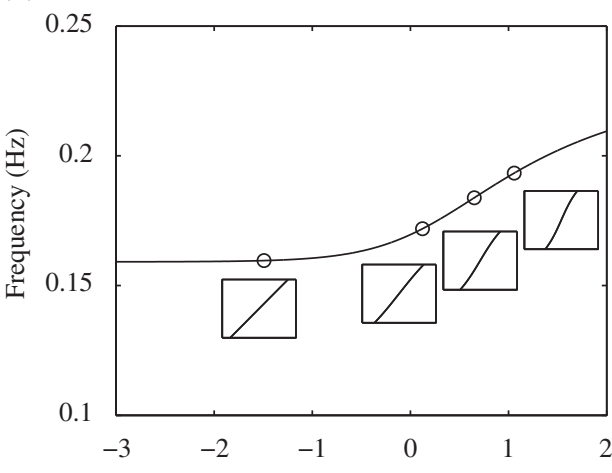

(b)

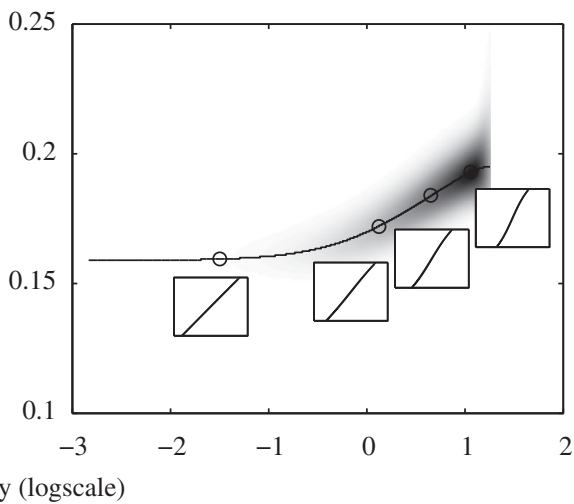

Fig. 21. Frequency-energy plot of the in-phase NNM of the 2DOF system. (a) Theoretical FEP computed by means of the numerical algorithm from the undamped system (2). (b) FEP calculated directly from the time series of the free damped response of system (10) using the CWT. The solid line is the ridge of the transform. 


\section{Numerical experiments of a nonlinear beam}

The proposed methodology is now demonstrated using simulated experiments of an existing nonlinear beam. This structure was used as a benchmark for nonlinear system identification during the European action COST F3 [41] and consists of a cantilever beam with geometrical nonlinearity. The finite element model considered herein is represented in Fig. 22. The main beam is modeled with 14 beam elements and the thin part with three beam elements. An additional rotational stiffness is used to model the junction between the two beams. As shown in [42,43], the nonlinear behavior of the thin beam can be modeled using a grounded cubic spring at the junction of the main and the thin beams. The cubic term takes the geometrical stiffening effect of the thin part into account. The geometrical and mechanical properties of the structure are listed in Tables 1 and 2.

\subsection{Theoretical modal analysis}

The NNMs of the underlying conservative system are computed using the numerical algorithm mentioned previously [22]. The theoretical FEPs of the first and second NNMs are represented in Figs. 23 and 24, respectively. The backbone of the plots corresponds to fundamental synchronous NNM motions. The NNM frequency undergoes a strong increase with increasing energy levels, which reveals the hardening behavior of the cubic stiffness nonlinearity. The evolution with energy of the modal shapes of the main beam, expressed in terms of displacement amplitudes, is also superposed. The FEPs highlight the presence of two other branches, termed tongues, emanating from the NNM backbone. These tongues reveal the existence of internal resonances. For instance, a 9:1 internal resonance between the first and third NNMs appears on the tongue in Fig. 23. As mentioned previously, this type of motions is addressed in [21] and is beyond the scope of this paper.

\subsection{Experimental modal analysis}

The proposed approach for EMA is now utilized. In this context, Newmark's numerical time integration scheme [36] is used. Linear proportional damping is considered to model dissipative forces in the structure. The damping matrix $\mathbf{C}$ is given by

$$
\mathbf{C}=3 \times 10^{-7} \mathbf{K}+5 \mathbf{M}
$$

which corresponds to moderate damping; for instance, the modal damping ratio is equal to 1.28 percent for the first linear normal mode. We note that the identified modal damping ratio is much smaller for the existing beam. Nevertheless, damping is deliberately chosen larger in order to ensure the applicability of the methodology to moderately damped structures.

\subsubsection{NNM force appropriation procedure}

Imperfect force appropriation: From a practical viewpoint, it is useful to study the quality of imperfect force appropriation consisting of a single-point mono-harmonic excitation, i.e., using a single shaker with no harmonics of the fundamental

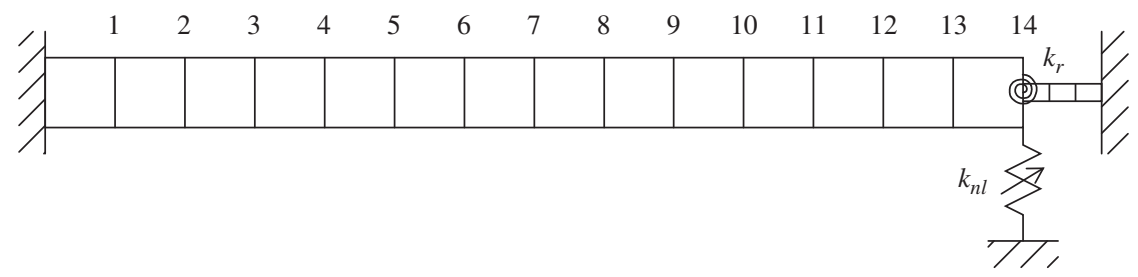

Fig. 22. Finite element model of the nonlinear beam.

Table 1

Geometrical properties of the nonlinear beam.

\begin{tabular}{llll}
\hline & Length $(\mathrm{m})$ & Width (m) \\
\hline Main beam & 0.7 & 0.014 & 0.014 \\
Thin beam part & 0.04 & 0.014 & 0.0005 \\
\hline
\end{tabular}

Table 2

Mechanical properties of the nonlinear beam.

\begin{tabular}{|c|c|c|}
\hline Young's modulus $\left(\mathrm{N} / \mathrm{m}^{2}\right)$ & Density $\left(\mathrm{kg} / \mathrm{m}^{3}\right)$ & Nonlinear coeff. $k_{\mathrm{nl}}\left(\mathrm{N} / \mathrm{m}^{3}\right)$ \\
\hline $2.05 \times 10^{11}$ & 7800 & $8 \times 10^{9}$ \\
\hline
\end{tabular}




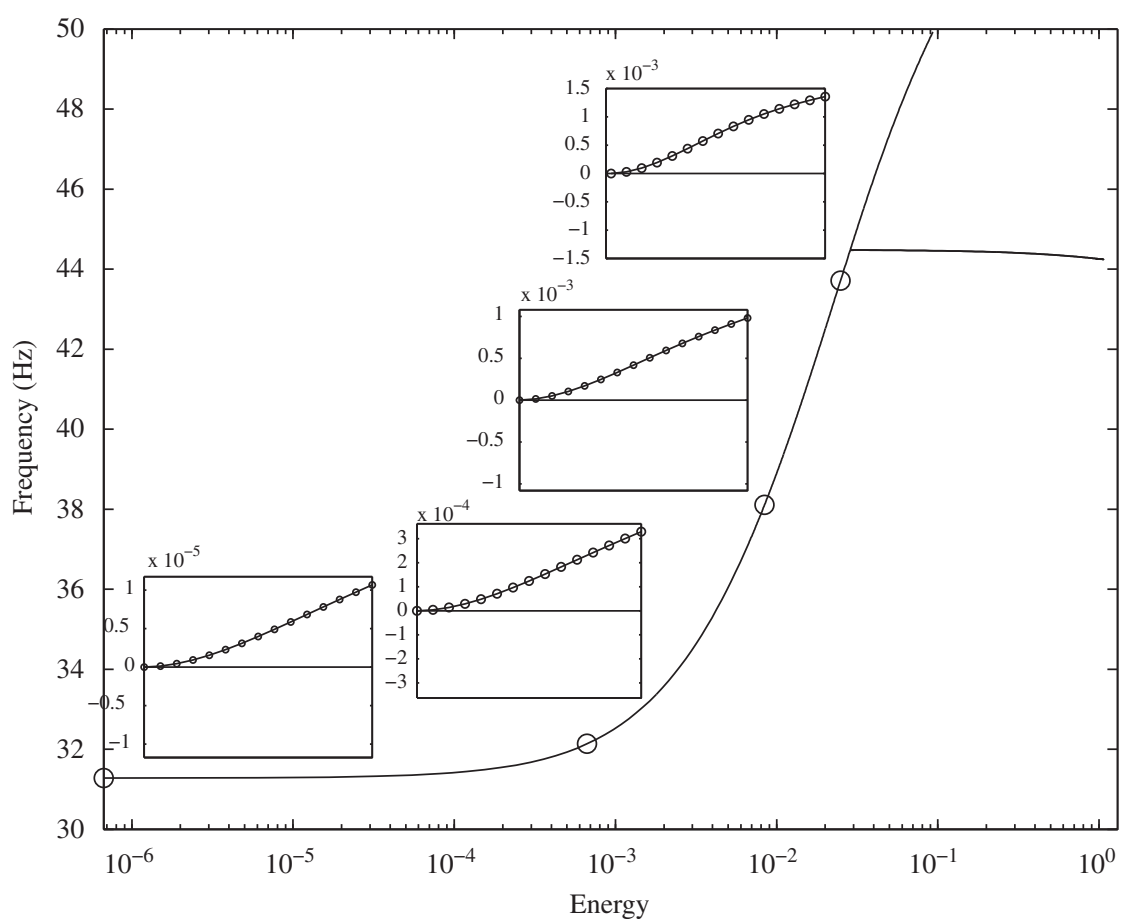

Fig. 23. Theoretical frequency-energy plot of the first undamped NNM of the nonlinear beam computed using the numerical algorithm. The NNM shapes (displacement amplitudes of the main beam) for four energy levels are inset.

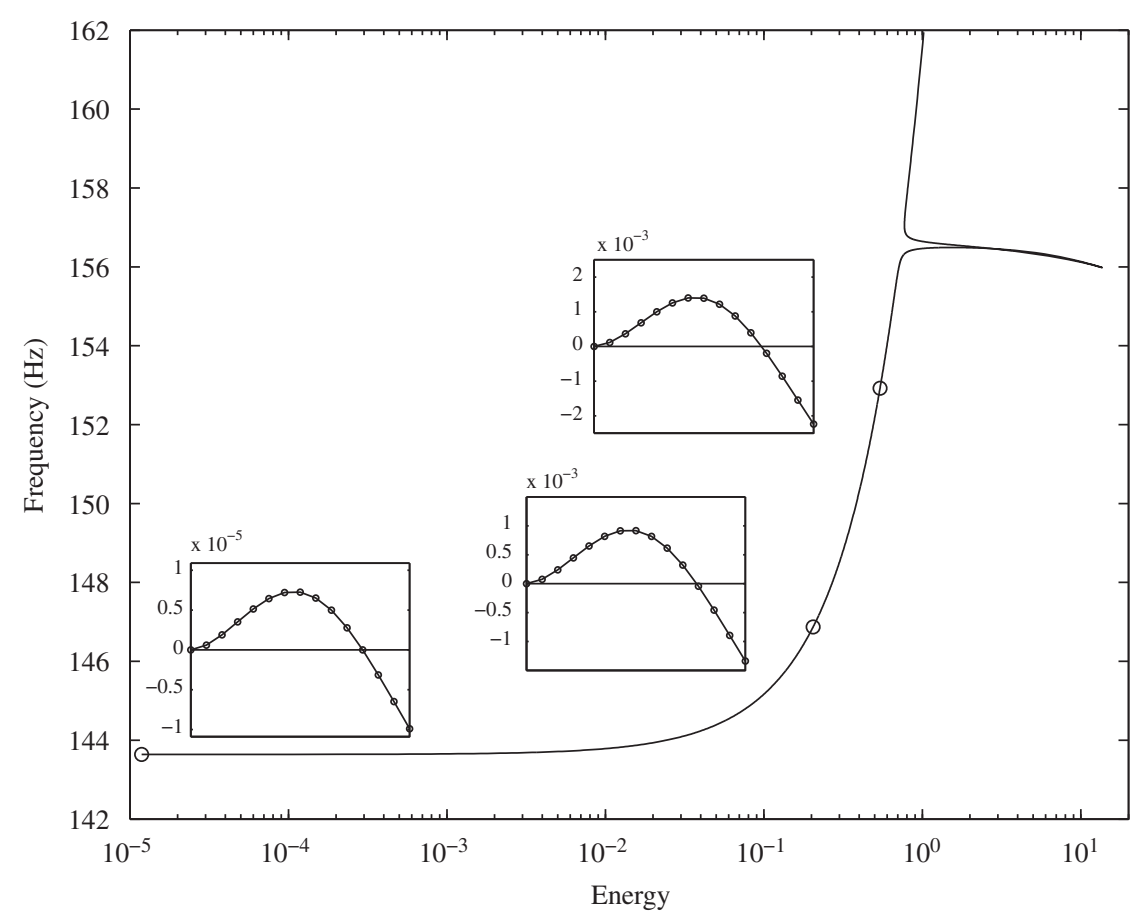

Fig. 24. Theoretical frequency-energy plot of the second undamped NNM of the nonlinear beam computed using the numerical algorithm. The NNM shapes (displacement amplitudes of the main beam) for four energy levels are inset.

frequency. The harmonic force $p(t)=F \sin (\omega t)$ is applied to node 4 of the main beam (see Fig. 22). The nonlinear forced frequency responses of the damped system close to the first resonance are numerically determined. They are represented in terms of displacement amplitudes for increasing forcing amplitudes $F$ in Fig. 25. The backbone of the undamped NNM of 

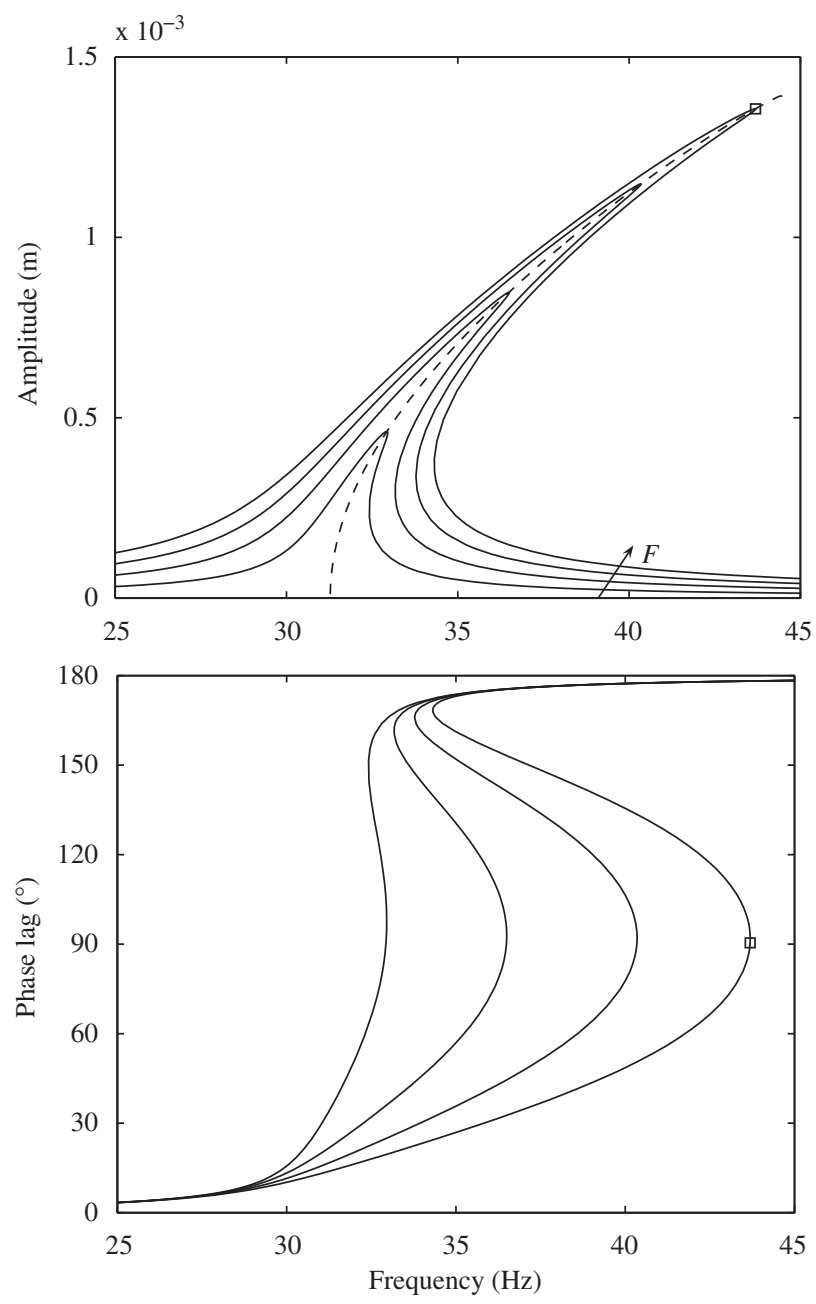

Fig. 25. Nonlinear forced frequency responses of the damped nonlinear beam close to the first resonant frequency (four different forcing amplitudes: 1,2 , $3,4 \mathrm{~N}$ ). The dashed line is the backbone of the first undamped NNM (computed by means of the numerical algorithm). Displacement at the tip of the main beam (node 14); top plot: amplitude, bottom: phase lag with respect to the excitation force.

(a)

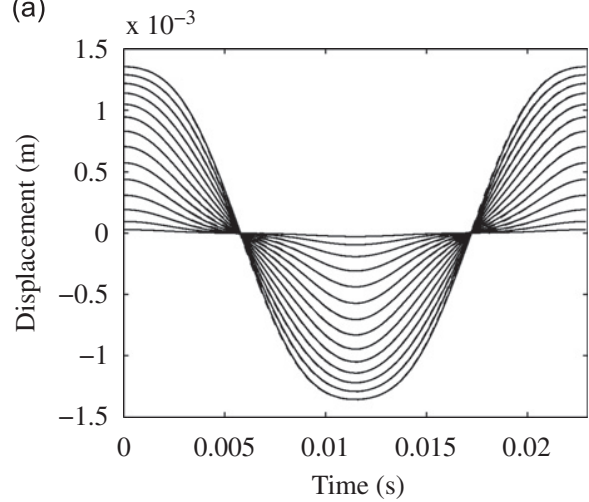

(b)

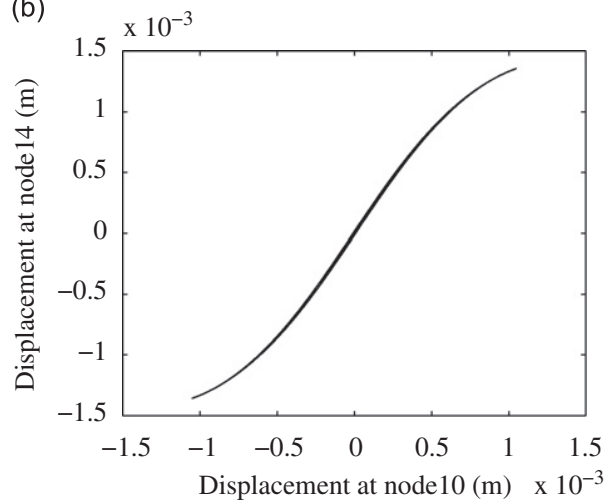

Fig. 26. Forced response (- of the damped nonlinear beam resulting from the imperfect force appropriation (for $F=4 \mathrm{~N}$ and marked by a square in Fig. 25) and the corresponding undamped NNM motion (---). (a) Time series of the displacements of the main beam. (b) Motion in the configuration space composed of the displacements at nodes 10 and 14 . 


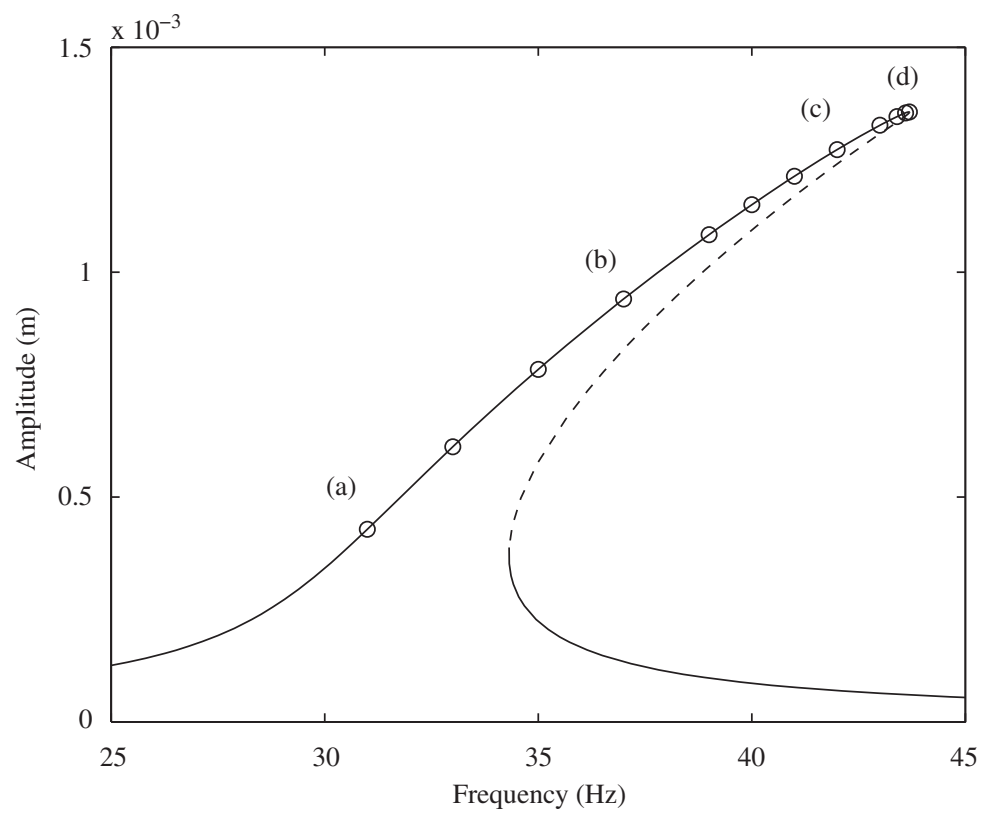

(a)

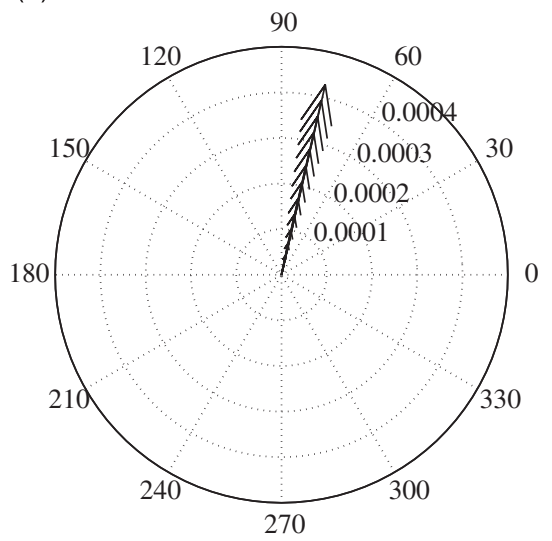

(c)

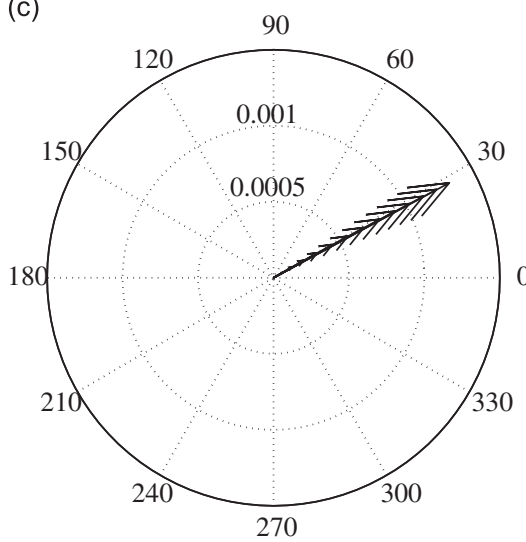

(b)

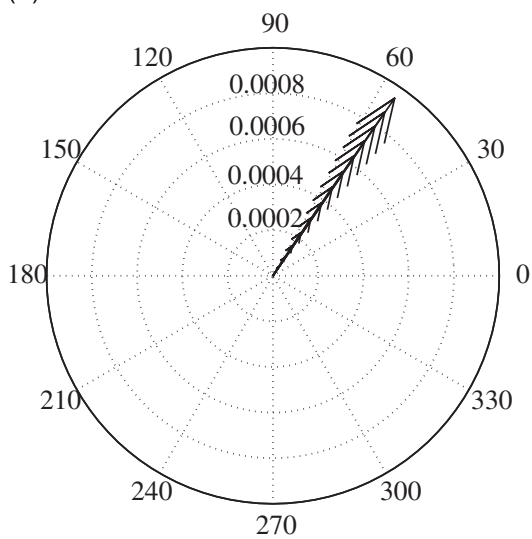

(d)

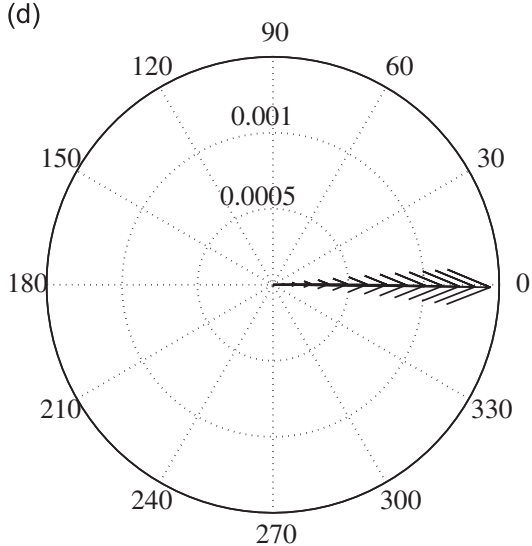

Fig. 27. Stepped sine excitation procedure for carrying out the NNM force appropriation $(F=4 \mathrm{~N})$ of the damped nonlinear beam. Top plot: responses along the branch close to the first resonance are marked by circles. Bottom plots: phase scatter diagrams of the complex Fourier coefficients of the displacements corresponding to the fundamental frequency for the responses (a), (b), (c) and (d). 
Fig. 23, expressed in terms of amplitude, is also superposed. Fig. 26 shows the forced damped response close to the resonance for $F=4 \mathrm{~N}$ (marked by a square in Fig. 25) and the corresponding undamped NNM motion at the same frequency. Results similar to those of Section 3.2 are obtained.

- The phase lag quadrature criterion is fulfilled close to resonant frequencies.

- Forced responses at resonance occur in the neighborhood of NNMs.

- Imperfect appropriation can isolate the NNM of interest (the beam has well-separated modes).

These findings also hold for the second beam NNM.

Practical realization of force appropriation: An assumption made throughout this paper is that the forced response at resonance can be reached (i.e., no instable, quasi-periodic and chaotic motions). Unlike the linear case, nonlinear systems possess coexisting stable solutions with their own domains of attraction. A procedure relying on stepped sine frequency response measurements is examined herein to study the practical feasibility of the force appropriation methodology for nonlinear structures. It gradually changes the frequency of the excitation $p(t)=F \sin (\omega t)$ to track the change of the forced frequency responses until the NNM force appropriation is achieved.

From the rest position, the structure is forced at the natural frequency of the undamped linear normal mode. After some transients, steady-state periodic forced response is obtained. Depending on the nonlinearities, the excitation frequency is then gradually increased or decreased to approach phase resonance. The phase lag indicator is continuously monitored during this process to assess the quality of the NNM force appropriation. The process is carried out until sufficiently good approximation of the phase quadrature criterion is achieved. In view of the basins of attraction of the coexisting forced responses, this procedure needs to adapt the frequency increments carefully. While quite large increments are initially suitable, smaller changes of the excitation frequency are required near the resonance to remain on the frequency response branch of interest. This stepped excitation frequency procedure is illustrated in Fig. 27 for a constant force amplitude
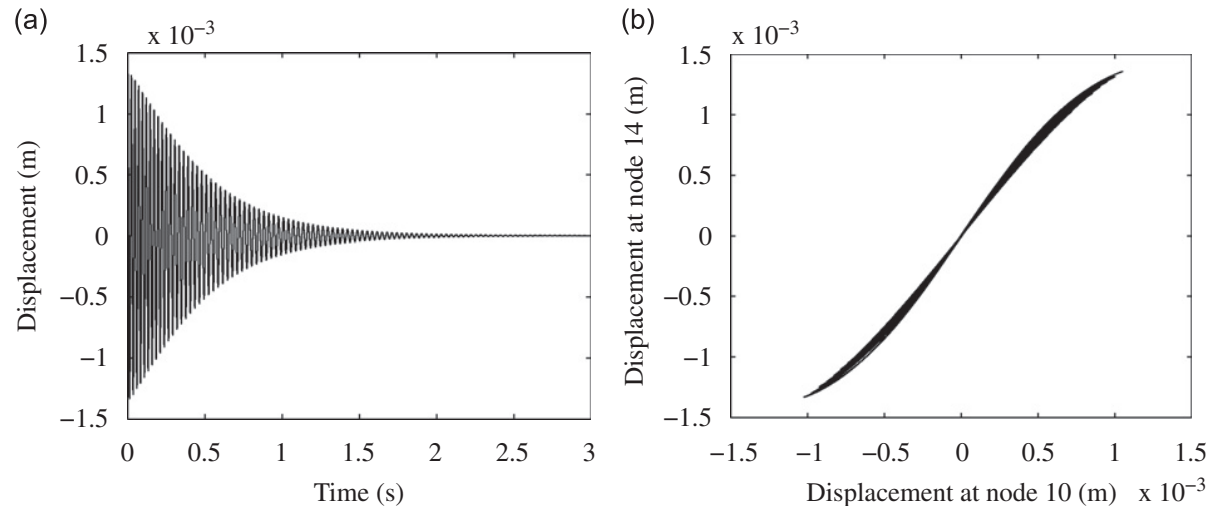

Fig. 28. Free response of the damped nonlinear beam initiated from the imperfect appropriated forced response represented in Fig. 26. (a) Time series of the displacement at the tip of the main beam (node 14). (b) Motion in the configuration space composed of the displacements at nodes 10 and 14.

(a)

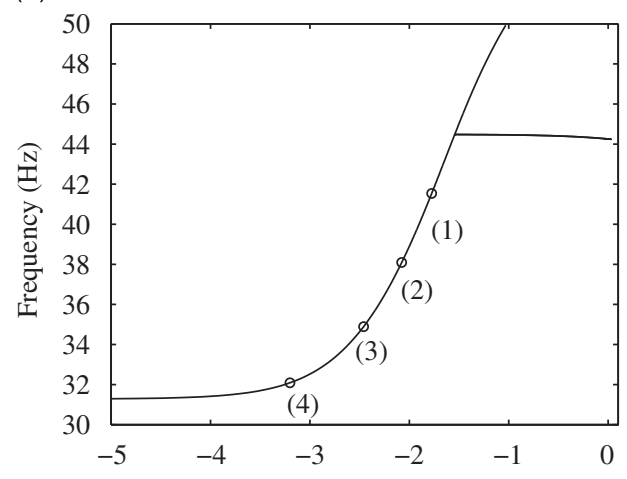

(b)

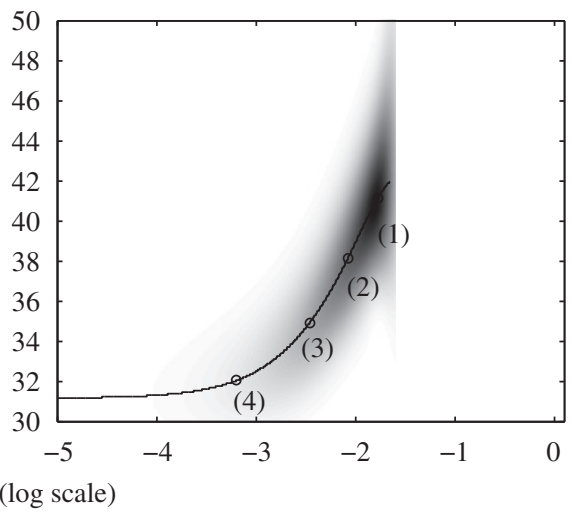

Fig. 29. Frequency-energy plot of the first NNM of the nonlinear beam. (a) Theoretical FEP. (b) FEP calculated from the time series of the free damped response using the CWT. The solid line is the ridge of the transform. 
$F=4 \mathrm{~N}$. The frequency responses obtained through this sequential process are represented by circles. The evolution of the phase response is also shown for four specific frequencies: the complex Fourier coefficients of the displacement responses corresponding to the fundamental frequency are illustrated. Since the excitation force $p(t)=F \sin (\omega t)$ is characterized by a phase of $90^{\circ}$, the NNM force appropriation is achieved when the phase of the displacements tends to $0^{\circ}$. This is performed for a frequency of $43.6 \mathrm{~Hz}$, which corresponds to point (d) in Fig. 27. The imperfect appropriated response of Fig. 26 is then reached, and the NNM is therefore isolated. The second fundamental NNM may be appropriated in the same way.

\subsubsection{NNM free decay identification}

Now that an NNM vibrates in isolation, its energy dependence is determined by turning off the excitation and tracking the single-NNM free damped response. The resulting response at the main beam tip is depicted in Fig. 28 . The dynamics closely follows the corresponding undamped NNM.

(a)
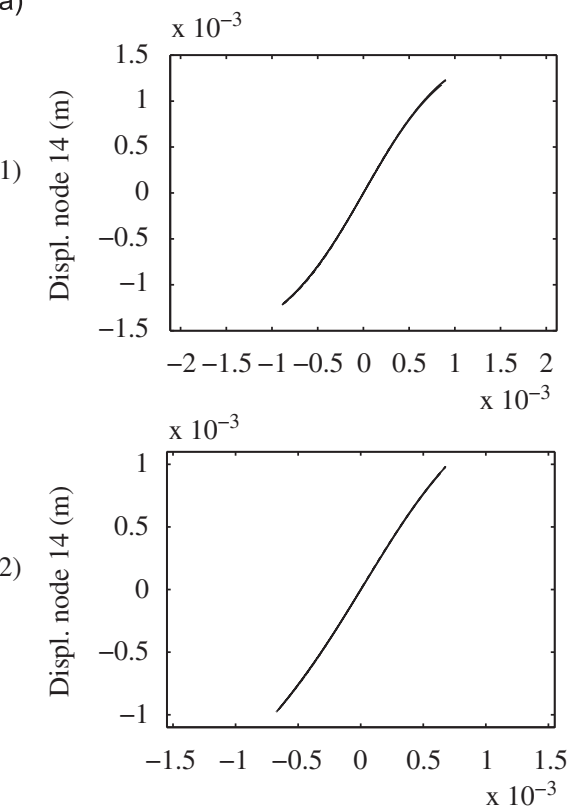

(b)
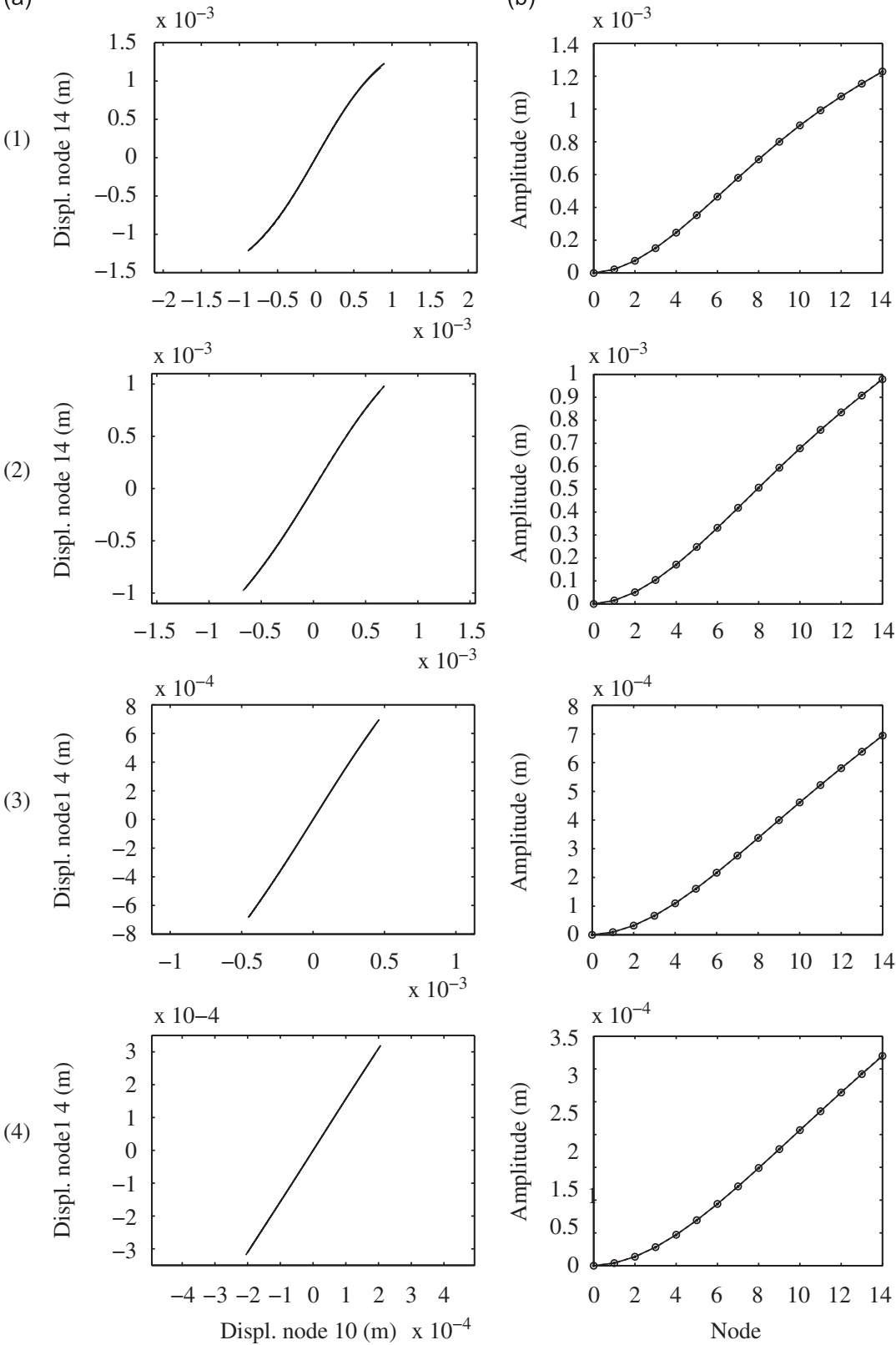

Fig. 30. Modal curves and modal shapes of the first NNM of the nonlinear beam for decreasing energy levels marked in Fig. 29 by circles (1), (2), (3) and (4). (a) Modal curves in the configuration space composed of the displacements at nodes 10 and 14. (b) Modal shapes composed of the displacement amplitudes of the main beam. The theoretical NNM motions are also superposed using dashed lines. 
(a)

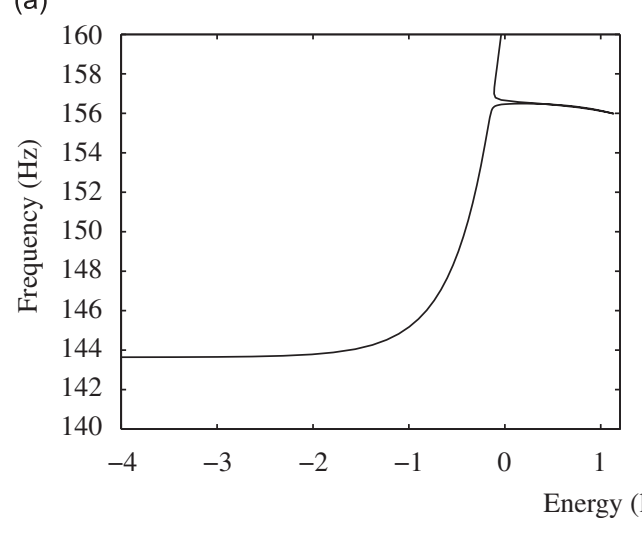

(b)

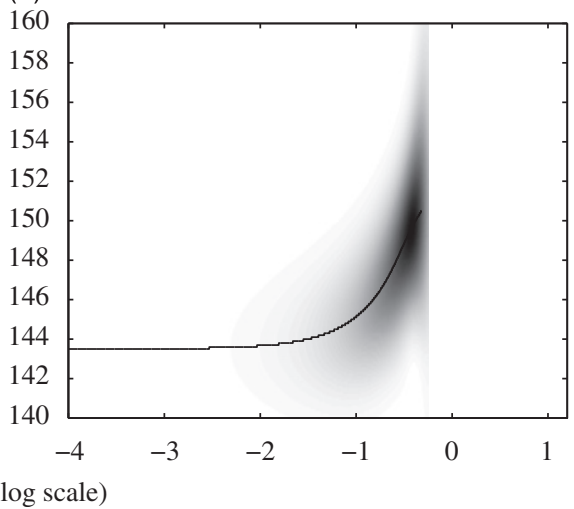

Fig. 31. Frequency-energy plot of the second NNM of the nonlinear beam. (a) Theoretical FEP. (b) FEP calculated from the time series of the free damped response using the CWT. The solid line is the ridge of the transform.

The FEP determined from the numerical experiments and computed through the CWT is illustrated in Fig. 29. The ridge of the transform provides the corresponding backbone. For comparison, the theoretical FEP computed from the conservative equations of motion is also represented. Apart from the CWT edge effects, a very good agreement is obtained between the two backbones. The relative error of the frequency is lower than 0.2 percent for the complete energy range.

The modal curves extracted from the time series at different energy levels (marked by circles in Fig. 29) are displayed in Fig. 30. The left plots represent the motions in a two-dimensional projection of the configuration space while the right plots depict the modal shapes. The theoretical undamped NNM motions are superposed using dashed lines. The theoretical modal curves and those extracted from the time series agree to the point where the motions cannot be distinguished. The MSE between the time series is consistently lower than 0.1 percent.

Similar results are obtained for the second NNM. For illustration, the FEPs are depicted in Fig. 31.

\section{Conclusion and future work}

Realizing that nonlinearity is a frequent occurrence in engineering structures and that linear EMA is of limited usefulness in this context, the present paper is an attempt to develop nonlinear EMA by targeting the extraction of NNMs from time series.

Because modal superposition is no longer valid, dynamic testing of nonlinear structures is realized through a nonlinear phase resonance method, which relies on the extension of the phase lag quadrature criterion. Specifically, if the forced response across the structure is a monophase periodic motion in quadrature with the excitation, an NNM vibrates in isolation. Once the NNM appropriation is achieved, the complete frequency-energy dependence of that nonlinear mode can be identified during the free decay response using time-frequency analysis. Eventually, an experimental FEP for one specific NNM can be obtained, and the procedure can be applied for all NNMs of interest. To relate the NNMs of the underlying undamped system to those extracted from the experimental data, the procedure assumes moderately damped systems possessing elastic nonlinearities.

This two-step methodology paves the way for a practical nonlinear analog of EMA, which may be applied to strongly nonlinear systems. For instance, it can certainly be a solid basis for extending standard ground vibration testing to nonlinear aircrafts. Through the combination of EMA with theoretical modal analysis, finite element model updating and validation of nonlinear structures are also within reach.

The objective of this paper was to lay down the foundations for a rigorous experimental identification of NNMs. However, several issues still need to be addressed before the methodology can be applied to large-scale, real-life structures. Unlike LNMs, which are neutrally stable, NNMs can be stable or unstable, which may complicate their practical realization. NNMs also possess basins of attraction which may shrink or expand along the backbone branch of the FEP. Sensitivity to measurement noise and uncertainty might therefore render nonlinear force appropriation more challenging than its linear counterpart. Experimental demonstration of these results is the next logical step and is currently under way using the existing nonlinear beam $[42,43]$.

\section{References}

[1] D.J. Ewins, A future for experimental structural dynamics, Proceedings of the International Seminar on Modal Analysis (ISMA), Leuven, Belgium, 2006

[2] J.S. Juang, R.S. Pappa, An eigensystem realization algorithm for modal parameter identification and model reduction, AIAA Journal of Guidance, Control and Dynamics 12 (1985) 620-627. 
[3] P. Van Overschee, B.L.R. De Moor, Subspace Identification for Linear Systems: Theory, Implementation, Applications, Kluwer Academic Publishers, Dordrecht, 1996

[4] B. Peeters, H. Van Der Auweraer, P. Guillaume, The polymax frequency domain method: a new standard for modal parameter estimation, Shock and Vibration 11 (2004) 395-409.

[5] R.J. Allemang, D.L. Brown, A unified matrix polynomial approach to modal identification, Journal of Sound and Vibration 211 (3) (1998) $301-322$.

[6] R.J. Allemang, A.W. Phillips, The unified matrix polynomial approach to understanding modal parameter estimation: an update, Proceedings of the International Seminar on Modal Analysis (ISMA), Leuven, Belgium, 2004.

[7] G. Benini, J.E. Cooper, J.R. Wright, Quantification of aircraft non-linearities from flight flutter test data, Proceedings of the International Seminar on Modal Analysis (ISMA), Leuven, Belgium, 2004.

[8] K. Carney, I. Yunis, K. Smith, C.Y. Peng, Nonlinear dynamic behavior in the cassini spacecraft modal survey, Proceedings of the International Modal Analysis Conference (IMAC), Orlando, USA, 1997.

[9] N. Okuizumi, M.C. Natori, Nonlinear vibrations of a satellite truss structure with gaps. Proceedings of the 45th AIAA Structures, Structural Dynamics E Materials Conference, Palm Springs, USA, 2004.

[10] G. Kerschen, K. Worden, A.F. Vakakis, J.C. Golinval, Past, present and future of nonlinear system identification in structural dynamics, Mechanical Systems and Signal Processing 20 (3) (2006) 505-592.

[11] P.A. Atkins, J.R. Wright, K. Worden, An extension of force appropriation to the identification of non-linear multi-degree of freedom systems, Journal of Sound and Vibration 237 (1) (2000) 23-43.

[12] M.F. Platten, J.R. Wright, G. Dimitriadis, J.E. Cooper, Identification of multi-degree of freedom non-linear systems using an extended modal space model, Mechanical Systems and Signal Processing 23 (1) (2009) 8-29.

[13] D. Goge, U. Fullekrug, M. Sinapius, M. Link, L. Gaul, Advanced test strategy for identification and characterization of nonlinearities of aerospace structures, AIAA Journal 43 (5) (2005) 974-986.

[14] W. Szemplinska-Stupnicka, The modified single mode method in the investigations of the resonant vibrations of non-linear systems, Journal of Sound and Vibration 63 (4) (1979) 475-489.

[15] W. Szemplinska-Stupnicka, Non-linear normal modes and the generalized Ritz method in the problems of vibrations of non-linear elastic continuous systems, International Journal of Non-linear Mechanics 18 (2) (1983) 149-165.

[16] S. Setio, H.D. Setio, L. Jezequel, A method of non-linear modal identification from frequency response tests, Journal of Sound and Vibration 158 (3) (1992) 497-515.

[17] S. Setio, H.D. Setio, L. Jezequel, Modal analysis of nonlinear multi-degree-of-freedom structures, The International Journal of Analytical and Experimental Modal Analysis 7 (2) (1992) 75-93.

[18] Y.H. Chong, M. Imregun, Development and application of a nonlinear modal analysis technique for mdof systems, Journal of Vibration and Control 7 (2001) 167-179.

[19] C. Gibert, Fitting measured frequency response using non-linear modes, Mechanical Systems and Signal Processing 17 (1) (2003) $211-218$.

[20] A.F. Vakakis, L.I. Manevitch, Y.V. Mikhlin, V.N. Pilipchuk, A.A. Zevin, Normal Modes and Localization in Nonlinear Systems, Wiley Series in Nonlinear Science, John Wiley \& Sons, New York, 1996.

[21] G. Kerschen, M. Peeters, J.C. Golinval, A.F. Vakakis, Nonlinear normal modes, part I: a useful framework for the structural dynamicist, Mechanical Systems and Signal Processing 23 (1) (2009) 170-194.

[22] M. Peeters, R. Viguié, G. Sérandour, G. Kerschen, J.C. Golinval, Nonlinear normal modes, part II: toward a practical computation using numerical continuation techniques, Mechanical Systems and Signal Processing 23 (1) (2009) 195-216.

[23] D. Goge, M. Boswald, U. Fullekrug, P. Lubrina, Ground vibration testing of large aircraft-state-of-the-art and future perspectives, Proceedings of the 25th International Modal Analysis Conference, Orlando, USA, 2007.

[24] B. Peeters, W. Hendricx, J. Debille, H. Climent, Modern solutions for ground vibration testing of large aircraft, Sound and Vibration 43 (2009) 8-15.

[25] R.M. Rosenberg, Normal modes of nonlinear dual-mode systems, Journal of Applied Mechanics 27 (1960) 263-268.

[26] R.M. Rosenberg, The normal modes of nonlinear n-degree-of-freedom systems, Journal of Applied Mechanics 30 (1) (1962) 7-14.

[27] R.M. Rosenberg, On nonlinear vibrations of systems with many degrees of freedom, Advances in Applied Mechanics 242 (9) (1966) 155-242.

[28] B. Fraeijs de Veubeke, A variational approach to pure mode excitation using characteristic phase lag theory, Technical Report, 1956.

[29] J.R. Wright, J.E. Cooper, M.J. Desforges, Normal-mode force appropriation-theory and application, Mechanical Systems and Signal Processing 13 (2) (1999) 217-240.

[30] M. Degener, Ground vibration testing for validation of large aircraft structural dynamics. Proceedings of the International Forum on Aeroelasticity and Structural Dynamics, Manchester, UK, 1995.

[31] M. Degener, J. Gschwilm, S.P. Lopriore, R.S. Capitanio, V.E. Hill, P.W. Johnston, Vibration tests for dynamic verification and qualification of the ppf/envisat-1 satellite, Proceedings of the 3rd International Symposium on Environmental Testing for Space Programmes-ESA SP-408, Noordwijk, The Netherlands, 1997, pp. 83-90.

[32] M. Degener, Experiences in large satellite modal survey testing, Proceedings of the European Conference on Spacecraft Structures, Materials and Mechanical Testing-ESA SP-428, Braunschweig, Germany, 1998, pp. 659-664.

[33] S.W. Shaw, C. Pierre, Normal modes for nonlinear vibratory systems, Journal of Sound and Vibration 164 (1) (1993) 85-124.

[34] A.F. Vakakis, O.V. Gendelman, L.A. Bergman, D.M. McFarland, G. Kerschen, Y.S. Lee, Nonlinear Targeted Energy Transfer in Mechanical and Structural Systems, 2009.

[35] P. Panagopoulos, F. Georgiades, S. Tsakirtzis, A.F. Vakakis, L.A. Bergman, Multi-scaled analysis of the damped dynamics of an elastic rod with an essentially nonlinear end attachment, International Journal of Solids and Structures 44 (2007) 6256-6278.

[36] M. Géradin, D. Rixen, Mechanical Vibrations: Theory and Application to Structural Dynamics, Wiley, Chichester, 1994.

[37] T.P. Le, P. Argoul, Continuous wavelet transform for modal identification using free decay response, Journal of Sound and Vibration 277 (1-2) (2004) $73-100$.

[38] L. Garibaldi, M. Ruzzene, A. Fasana, B.A.D. Piombo, Identification of non linear damping mechanisms using the wavelet transform, Mecanique Industrielle Et Materiaux 51 (2) (1998) 92-94.

[39] W.J. Staszewski, Identification of non-linear systems using multi-scale ridges and skeletons of the wavelet transform, Journal of Sound and Vibration 214 (4) (1998) 639-658.

[40] S.G. Mallat, A Wavelet Tour of Signal Processing, second ed., Academic Press, San Diego, 1999

[41] F. Thouverez, Presentation of the ECL benchmark, Mechanical Systems and Signal Processing 17 (1) (2003) 195-202.

[42] G. Kerschen, V. Lenaerts, J.C. Golinval, Identification of a continuous structure with a geometrical non-linearity. Part I: conditioned reverse path method, Journal of Sound and Vibration 262 (4) (2003) 889-906.

[43] V. Lenaerts, G. Kerschen, J.C. Golinval, Identification of a continuous structure with a geometrical non-linearity. Part II: proper orthogonal decomposition, Journal of Sound and Vibration 262 (4) (2003) 907-919. 